\title{
Group Strategies and Independence
}

\author{
Ming Xu
}

\begin{abstract}
We expand Belnap's general theory of strategies for individual agents to a theory of strategies for multiple agents and groups of agents, and propose a way of applying strategies to deal with future outcomes at the border of a strategy field. Based on this theory, we provide a preliminary analysis on distinguishability and independence, as a preparation for a general notion of dominance in the decisiontheoretical approach to deontic logic.
\end{abstract}

Based on branching time and a theory of agents and choices, Belnap has developed a general theory of strategies in Belnap (1996b) and Belnap et al. (2001). ${ }^{1}$ A simple form of this theory identifies a strategy for an agent with a partial function from moments to the choices available for the agent at those moments, which is found useful by different authors in conceptual analysis and technical development concerning "strategic acts". 2 Horty develops a simpler but similar theory of strategies in Horty (2001), and applies it to his study of "strategic acts" and "strategic oughts". The work presented here concerns both "strategic acts" and "strategic oughts", perhaps with an emphasis on the latter in the background. This paper is the first step of a project to connect Belnap's theory and the decision-theoretical approach to deontic

\footnotetext{
${ }^{1}$ I would like to give thanks to Nuel Belnap for his comments and encouragements, and to Yan Zhang for several discussions and for catching errors in early drafts of this paper. For the theory of branching time, see Prior (1967) and Thomason (1970,1984); and for the theory of agents and choices, see, e.g., Belnap (1991, 1996a) and Belnap et al. (2001)

${ }^{2}$ For example, Belnap shows that whenever a doing takes place, there exists a strategy of refraining from that doing (see chapter 13 of Belnap et al. 2001), Müller applies this theory of strategies to deal with continuous actions in Müller (2005), and Broersen and his colleagues apply this theory in their work to extend alternating-time temporal logic in Broersen et al. (2006).
}

\section{Xu ( $\square)$}

Department of Philosophy, Wuhan University, Wuhan 430072, People's Republic of China e-mail: mingxu01@hotmail.com 
logic developed in Horty (2001), in a setting involving multiple agents and groups of agents. ${ }^{3}$

In the decision-theoretical approach to deontic logic, what an agent ought to do is taken to be determined by the result of an evaluation of what she can do against background situations or conditions in the form of a partition. If one action is taken to be better than another under each such background condition, it is then inferred to be better than the other unconditionally, or to "dominate" the other, as is often described. ${ }^{4}$ The background conditions, however, are required to be independent of the actions being evaluated. This independence requirement is essential, without which the inference is evidently flawed. ${ }^{5}$ In Horty (2001), Horty takes the notion of independence here to be causal independence, and presents the background conditions, when evaluating actions of an agent or a group, as what other agents may do at the same time. In other words, actions at the same time by different agents are taken to be independent of each other.

This approach to deontic logic is continued in Kooi and Tamminga (2008) and later in Tamminga (2013), with a notion of relative dominance and a closer relation to game theory. It has so far been limited, nevertheless, to either single-step group actions, or strategies of a single agent while other agents are assumed absent. The reason for such limitation is, I think, that it is not clear how to deal with the independence requirement in a setting involving actions at different moments by different agents, as Horty seems to suggest in Horty (2001). This paper examines strategies for different groups and some relations between them, based on which we develop a notion of independence of strategies for different groups, by way of an analysis of distinguishability and inactivity. We provide some results concerning independence (in Sect.9), including a characterization of independence in terms of a set-theoretical relation between groups of agents (Theorems 9.6 and 9.10).

Section 1 briefly presents the background theories of branching time, agents and choices, and Sects. 2 and 3 present our notions of outcomes and fields with outcomes at their "borders". In Sects.4-6, we discuss group strategies with respect to future outcomes and various related notions. Finally we present a preliminary analysis of the notions of distinguishability, inactivity and independence in Sects.7-9, as a preparation for a future work on dominance.

\footnotetext{
${ }^{3}$ Belnap's theory of strategies may also be applied to other approaches to deontic logic. For example, Belnap (1996b) shows the connection between his theory of strategies and Thomason's theory of ought kinematics (Thomason 1984).

4 The kind of inference applied here is sometime called the "sure-thing principle" (see Savage 1954).

${ }^{5}$ See discussions in, e.g., Thomason and Horty (1996) and Horty (2001).
} 


\section{Stit Frames}

In this section, we briefly present the basic notions in the semantic theory for stit, ${ }^{6}$ which constitute a general background for our theory concerning what agents may do relative to future outcomes. Let us start with the branching time theory developed by A. Prior and R. Thomason.

A tree-like frame is a pair $\langle T,<\rangle$, in which $T$ is a nonempty set, and $<$ is a strict partial ordering on $T$ (i.e., an irreflexive and transitive relation on $T$ ) satisfying the following conditions:

NBB : for all $x, y, z \in T$, if $y<x$ and $z<x$, either $y \leqslant z$ or $z \leqslant y$;

$\mathrm{HC}$ : for all $x, y \in T, z \leqslant x$ and $z \leqslant y$ for some $z \in T$;

where $x \leqslant y$ iff $x<y$ or $x=y$. The label NBB is for "no backward branching", and $\mathrm{HC}$ for "historical connection". 7

We call members of $T$ moments or points, for which we use $m, u, x, y, z$ etc., and call each maximal <-chain of moments in $T$ a history (in $\langle T,<\rangle$ ). We use $h, h^{\prime}$ etc. for histories and $H, H^{\prime}$ etc. for sets of them. In particular, we use $H_{T}$ for the set of all histories (in $\langle T,<\rangle$ ). Furthermore, we will apply the following notations and expressions, where $M \subseteq T, c$ is a chain (of points), and $x$ a point, in $T$ :

- $H_{\langle M\rangle}=\left\{h \in H_{T}: h \cap M \neq \varnothing\right\}$, histories passing through $M$;

- $H_{[c]}=\left\{h \in H_{T}: c \subseteq h\right\}$, histories passing completely through $c$;

- $H_{x}=\left\{h \in H_{T}: x \in h\right\}$, histories passing through $x$.

It is plain that $H_{x}=H_{[\{x\}]}=H_{\langle\{x\}\rangle}$. Sets of histories are compatible if their intersection is nonempty. It is easy to see that for all $x$ and $y$, if neither $x \leqslant y$ nor $y \leqslant x$, then no subset of $H_{x}$ is compatible with any subset of $H_{y}$.

A sequence $\langle T,<$, Agent, Choice $\rangle$ is a stit frame if $\langle T,<\rangle$ is a tree-like frame, Agent is a nonempty set of "agents", and Choice is a function that assigns to each $\alpha \in$ Agent and each $m \in T$ a partition Choice ${ }_{\alpha}^{m}$ of $H_{m}$ satisfying the following conditions:

NC : for each $K \in$ Choice $_{\alpha}^{m}$, each $h \in K$ and each $x \in h$, if $m<x$ then $H_{x} \subseteq K$;

\footnotetext{
${ }^{6}$ Stit, the acronym of "sees to it that", was taken to name a modal operator used in a rigorous philosophical theory of agency and action developed in a series of articles by Belnap, Perloff and their colleagues, which provides, among other things, formal semantics for sentences involving what agents do. The acronym was soon used to refer to the theory itself, and later to other theories as well that share similar principles, methods and logical tools. Stit theories were developed by a number of people in the late 1980s, and have now become a field to which many people have contributed their works. So I will just mention a few pieces of work, among many others, which basically started this field: Belnap and Perloff (1988), von Kutschera (1986) and Horty (1989). For detailed discussions in stit theories, see, e.g., Belnap et al. (2001).

${ }^{7}$ When $\langle T,<\rangle$ satisfies all conditions above for a tree-like frame except the condition $\mathrm{HC}$, we may call it a multi-tree-like frame. For the purpose of this paper, we will focus on structures based on treelike frames, but our discussions can easily be extended to similar structures based on multi-tree-like frames.
} 
IA : for each function $f$ that assigns to each $\beta \in$ Agent a member $f(\beta)$ of Choice $_{\beta}^{m}$, $\bigcap_{\beta \in \text { Agent }} f(\beta) \neq \varnothing$.

The label NC is for "no choice between undivided histories" and IA for "independence of agents". A function $f$ is a selection function at $m$ if $f(\beta) \in$ Choice $_{\beta}^{m}$ for each $\beta \in$ Agent. We will use Select ${ }_{m}$ for the set of all selection functions at $m$. Thus IA above can be restated as that $\bigcap_{\beta \in \text { Agent }} f(\beta) \neq \varnothing$ for each $f \in$ Select $_{m}$.

Let $\langle T,<$, Agent, Choice $\rangle$ be any stit frame. We call subsets of Agent groups (of agents), and use $\mathcal{E}, \mathcal{F}, \mathcal{G}$ etc. to range over them. For each $m \in T$ and each group $\mathcal{G}$, we use Choice $_{\mathcal{G}}^{m}$ for $\left\{\bigcap_{\alpha \in \mathcal{G}} f(\alpha): f \in\right.$ Select $\left._{m}\right\}\left(\right.$ Choice $\left._{\varnothing}^{m}=\left\{H_{m}\right\}\right),{ }^{8}$ call its members possible choices for $\mathcal{G}$ at $m$, and use $K, K^{\prime}$ etc. to range over them. A possible choice, or simply a choice, is a possible choice for a group at a point. A group $\mathcal{G}$ (or an agent $\alpha$ ) has vacuous choice at a point $m$ if Choice $_{\mathcal{G}}^{m}=\left\{H_{m}\right\}$ (Choice $\alpha_{\alpha}^{m}=\left\{H_{m}\right\}$ ). Provided that $h \in H_{m}$, we use Choice $_{\mathcal{G}}^{m}(h)$ for the unique member of Choice $_{\mathcal{G}}^{m}$ to which $h$ belongs. Finally, we let $\overline{\mathcal{G}}=$ Agent $-\mathcal{G}$ for each group $\mathcal{G}$. It is easy to verify the following by applying NC:

Fact 1.1. For each $\mathcal{G}$ and all $x, y \in h$ such that $y<x, H_{x} \subseteq$ Choice $_{\mathcal{G}}^{y}(h)$.

By this fact, we introduce the following notation: provided that $y<x$, we use Choice $_{\mathcal{G}}^{y}\left(H_{x}\right)$ for the unique $K \in$ Choice $_{\mathcal{G}}^{y}$ such that $H_{x} \subseteq K$.

\section{Outcomes}

In many cases, it is more convenient to use outcomes rather than histories for conceptual analysis or technical development. In this section we discuss a notion of outcome, derived from Xu (1997). We first present the notion in its original form and then convert it into a notion of history-outcome. Throughout this section and the next, we fix a tree-like frame $\langle T,<\rangle$, with respect to which our discussions are to be understood.

Our notion of outcomes presupposes the following: For each $x \in T$ and each $X \subseteq T, x \leqslant X(X \leqslant x, x<X$ or $X<x)$ iff $x \leqslant y(y \leqslant x, x<y, y<x)$ for every $y \in X$, and in such a case, we say that $x$ is a lower-bound (upper-bound,proper lower-bound, proper upper-bound) of $X$. A subset $X$ of $T$ is forward (backward) closed if for all $x, y \in T, x<y(y<x)$ and $x \in X$ only if $y \in X$. A past in $\langle T,<\rangle$ is a nonempty and properly upper-bounded set $p$ of moments that is backward closed. For each past $p, H_{[p]}$ is by definition $\left\{h \in H_{T}: p \subseteq h\right\}$, the set of all histories passing completely through $p$. For each properly upper-bounded nonempty chain $c$ of moments, we use $p_{c}$ for the smallest past including $c$, i.e., $p_{c}=\{x \in T$ :

\footnotetext{
${ }^{8}$ I do not mean to take the empty set as a group or an agent in the literal sense. The only reason why we call it a group is for technical convenience. One could exclude it from groups and add extra conditions in our technical discussions.
} 
$\exists y \in c(x \leqslant y)\}$. Thus for each $x \in T$ that is not maximal in $T, p_{\{x\}}$ is the past $\{y \in T: y \leqslant x\}$.

We want to add the notion of outcomes to the theories of strategies developed in Belnap (1996b) and Horty (2001) in order to extend their theories to deal with strategy-weighing relative to the values of future outcomes. An "outcome" can be reified either as a set of moments or as a set of histories, with a simple relation between them.

An outcome (in $\langle T,<\rangle$ ) is a nonempty and properly lower-bounded set $O$ of moments that is forward closed and historically connected in $O$, i.e., for all $x, y \in$ $O, z \leqslant x$ and $z \leqslant y$ for some $z \in O$. For each past $p$, an outcome at $p$ is an outcome $O$ such that $p$ is the set of all its proper lower-bounds, i.e., $p=\{x \in T: x<O\}$. For each nonempty chain $c$ of moments in $T$, an outcome at $c$ is an outcome at $p_{c}$, and for each $x \in T$, an outcome at $x$ is an outcome at $\{x\}$.

In Xu (1997), an outcome $O$ is paired with a past $p$ to form a "transition" $\langle p, O\rangle$, where $p<x$ for every $x \in O$, which is used to characterize a process or change from the state $p$ right before the process to the outcome state $O$ of the process. So an outcome $O$ marks the temporal "location" of the completion of a process in such a way that all histories overlapping $O$ are taken to be just those in which the process completes. For technical simplicity, we do not use the notion of transition explicitly in this paper. We apply its idea extensively, nevertheless. The following fact is easily verifiable.

Fact 2.1. Let $p$ be any past, and let $O$ be any outcome at $p$. Then for each history $h, h \cap O \neq \varnothing$ only if $h-p \subseteq O$.

Let $p$ be any past, and let $\sim_{p}$ be a relation between histories in $H_{[p]}$ such that for all $h, h^{\prime} \in H_{[p]}, h \sim_{p} h^{\prime}$ iff $x \in h \cap h^{\prime}$ for some $x>p$. It is easy to verify that $\sim_{p}$ is an equivalence relation. A history-outcome at $p$ is an equivalence class modulo $\sim_{p}$. A history-outcome at a properly upper-bounded nonempty chain $c$ (or at a nonmaximal point $m$ ) is a history-outcome at the past $p_{c}\left(p_{\{m\}}\right)$, and a history-outcome is a history-outcome at a past.

Proposition 2.2. For all history-outcomes $H$ and $H^{\prime}$, either $H \subseteq H^{\prime}$ or $H^{\prime} \subseteq H$ or $H \cap H^{\prime}=\varnothing$.

Proof. Let $H$ and $H^{\prime}$ be outcomes at $p$ and $p^{\prime}$ respectively, and suppose that $h_{0} \in$ $H \cap H^{\prime}$ and $h^{\prime} \in H^{\prime}-H$. It then suffices to let $h \in H$ and show that $h \sim_{p^{\prime}} h_{0}$, which implies that $h \in H^{\prime}$. Since $h_{0}, h \in H$ and $h_{0}, h^{\prime} \in H^{\prime}$, there are $x$ and $y$ such that $p<x \in h \cap h_{0}$ and $p^{\prime}<y \in h_{0} \cap h^{\prime}$. Because $h_{0} \in H$ and $h^{\prime} \notin H, h^{\prime} \chi_{p} h_{0}$, i.e.,

$$
p \nless z \text { for each } z \in h_{0} \cap h^{\prime} \text {. }
$$

Because $x, y \in h_{0}$, either $x<y$ or $y \leqslant x$. If $x<y$, then $p<y$ since $p<x$, and, since $y \in h_{0} \cap h^{\prime}, p \nless y$ by (1), a contradiction. It then follows that $y \leqslant x$, and then $p^{\prime}<x$, and hence $h \sim{ }_{p^{\prime}} h_{0}$. 
Now we have two kinds of outcomes, whose relation needs to be made clear. To help our discussion, let us refer to the kind of outcomes defined earlier as momentoutcomes.

Proposition 2.3. Let $p$ be a past, and let $f$ be a function on the set of all momentoutcomes at $p$ such that for each such outcome $O, f(O)=\left\{h \in H_{T}: O \cap h \neq \varnothing\right\}$. Then $f$ is a one-one correspondence between the set of all moment-outcomes at $p$ and the set of all history-outcomes at $p$.

Proof. For all $h, h^{\prime} \in f(O)$, there are $x \in O \cap h$ and $y \in O \cap h^{\prime}$, and then by the condition of historical connection on $O$, there is a $z \in O$ such that $z \leqslant x$ and $z \leqslant y$, and hence $z \in h \cap h^{\prime}$. Since $z \in O, p<z$, and hence $h \sim_{p} h^{\prime}$. It follows that $f(O)$ is included in an equivalence class modulo $\sim_{p}$. To see that it is itself an equivalence class modulo $\sim_{p}$, it suffices to suppose that $h \sim_{p} h^{\prime}$ with $h \in f(O)$, and show that $h^{\prime} \in f(O)$. By definition, $m \in h \cap h^{\prime}$ for an $m>p$. By Fact $2.1, h-p \subseteq O$, and then, since $m>p$ and $m \in h, m \in O$, and hence $h^{\prime} \in f(O)$.

Belnap and Horty use histories to define various notions in their study of strategies. It is then more convenient to use history-outcomes rather than moment-outcomes in our presentation to show a clear picture of the connection between our theory and theirs. By Proposition 2.3, the two kinds of outcomes are different notions of the same idea. ${ }^{9}$ From now on, when we speak simply of outcomes, we mean history-outcomes.

For each past $p$, we use Outcm $_{p}$ for the set of all outcomes at $p$, and for each properly upper-bounded nonempty chain $c$, we use $\mathrm{Outcm}_{c}$ for $\mathrm{Outcm}_{p_{c}}$, and, finally, for each non-maximal point $x$, we use $\operatorname{Outcm}_{x}$ for $\operatorname{Outcm}_{\{x\}}$. It is easy to see that each history $h$ in $H_{[p]}$ belongs to a unique outcome at $p$, and thus we use $\operatorname{Outcm}_{p}(h)$ for that outcome. Similarly, for each history $h$ passing completely through a properly upper-bounded nonempty chain $c$ or through a non-maximal point $x$, we will use $\operatorname{Outcm}_{c}(h)$ or $\operatorname{Outcm}_{x}(h)$ for the outcome at $c$ or $x$ to which $h$ belongs. It is routine to verify the following by applying relevant definitions.

Fact 2.4. Let $h$ be any history, let $\{x\}, c \subseteq h$, both of which are properly upperbounded, and let $c$ be nonempty. Then $c<x$ only if $H_{x} \subseteq \operatorname{Outcm}_{c}(h)$, and $c \nless x$ only if $\operatorname{Outcm}_{c}(h) \subseteq \operatorname{Outcm}_{x}(h)$.

Fact 2.5. Let $\langle T,<$, Agent, Choice $\rangle$ be a stit frame, let $\mathcal{G}$ be any group, and let $x \in h$, where $x$ is not a maximal point. Then $\operatorname{Outcm}_{x}(h) \subseteq$ Choice $_{\mathcal{G}}^{x}(h)$.

The converse of Fact 2.5 does not in general hold: a possible choice for any group (including Agent) at a moment may consist of several outcomes at the moment. How many outcomes can there be at a past $p$ without a maximum? The answer is that there

\footnotetext{
${ }^{9}$ There is nevertheless a shortcoming in a presentation using history-outcomes. Set-theoretically speaking, moment-outcomes at different moments are always different, while history-outcomes at different moments may turn out to be the same. For example, if $c$ is a nonempty segment of a history in which no histories split at any point, then history-outcomes at points in $c$ remain the same. For more discussions of the notion of moment-outcomes and its applications, see Xu (1997, 2010, 2012) and Brown (2008).
} 
may still be more than a single outcome at $p$ even though for all $h, h^{\prime} \in H_{[p]}, h \in$ Choice $e_{\text {Agent }}^{x}\left(h^{\prime}\right)$ for every $x \in p$, i.e., $h$ and $h^{\prime}$ are not distinguished by any possible choices at points in $p$. A stit frame $\langle T,<$, Agent, Choice $\rangle$ is agency determinate if for each past $p$ and each history $h$ passing completely through $p, \bigcap_{x \in p} \operatorname{Choice}_{\text {Agent }}^{x}(h)$ is a single outcome at $p$. In certain applications, agency determination or similar conditions are proposed to make the semantic structures ideal in some sense. Since the purpose of the current study is to provide a general theory, we will not include this condition for our general framework.

\section{Fields and Outcomes Bordering Fields}

An anti-chain (in $\langle T,<\rangle$ ) is a nonempty subset $i$ of $T$ such that for all $x, y \in i$, neither $x<y$ nor $y<x$. Let $i$ be any anti-chain in $\langle T,<\rangle$. $i$ intersects a history $h$ if $i \cap h \neq \varnothing$. If $i$ intersects $h, i \cap h$ is clearly a singleton, and in such a case, we use $m_{i, h}$ for the unique member of $i \cap h$.

A field is a nonempty subset $M$ of $T$. An anti-chain $i$ covers a field $M$ ( $i$ is a cover of $M$ ) if for each $x \in M, x \leqslant y$ for a $y \in i$ and $i$ intersects every $h \in H_{x}$ and $x \leqslant m_{i, h} \cdot{ }^{10} M$ is covered if it is covered by an anti-chain, and is properly covered if it is covered by an anti-chain $i$ such that $i \cap M=\varnothing$. A properly covered field has two roles in the current study. The first is to provide a background choice situation for our discussion of strategies, and the second is to constrain the so-called future outcomes that agents or groups may attain.

Covered fields may take various "shapes", and it is their "borders" in the future and the outcomes at the "borders" in which we are interested. A cover of a field guarantees the field to have a "border", and a proper cover even guarantees that there are outcomes everywhere along the "border". They are not accurate, nevertheless, in telling where exactly the "border" is, much less about the outcomes there; for they may contain points in the field as well as points far beyond the "border". We then have to find another way to talk about the outcomes at the "border" of a field.

Let $M$ be any field. $M$ is inward closed if for all $x, y, z \in T$ such that $x<$ $y<z$, if $x, z \in M$ then $y \in M$. We use $M^{+}$for the inward closure of $M$, i.e., $M^{+}=\{x \in T: \exists y, z \in M(y \leqslant x \leqslant z)\}$. A history $h$ passes across $M$ if $\varnothing \neq M \cap h<x \in h$ for some $x$. It is obvious that $h$ passes across $M$ only if $h \in H_{\langle M\rangle}$, but the converse does not hold in general. An outcome $H$ is an $M$ bordering outcome (or an outcome bordering $M$ ) if there is a history $h$ passing across $M^{+}$such that $H=$ Outcm $_{M^{+} \cap h}(h) .{ }^{11}$ For each field $M$, we will use $O u t c m B d r_{M}$

\footnotetext{
${ }^{10}$ When assuming the Axiom of Choice, the clause " $x \leqslant y$ for a $y \in i$ " is redundant.

${ }^{11}$ Let $h$ pass across $M$, i.e., $M \cap h<x \in h$ for an $x$. If $M$ is not inward closed, there may be a $y \in M$ such that $x<y \notin h$ and $H_{y} \subset H_{x} \subseteq \operatorname{Outcm}_{M \cap h}(h)$. The outcome $\operatorname{Outcm}_{M \cap h}(h)$ should not be taken to be bordering $M$, and to rule out such outcomes as $M$-bordering outcomes, we need to use $M^{+}$instead of $M$ in our definition. The definition of $M$-bordering outcomes in terms of moment-outcomes is simpler: $O$ is an $M$-bordering outcome if $O \cap M=\varnothing$ and $O$ is an outcome at a nonempty chain $c$ in $M^{+}\left(c \subseteq M^{+}\right)$.
} 
for the set of all $M$-bordering outcomes. It is easy to see that if a field $M$ is inward closed, then for each outcome $H, H \in \operatorname{OutcmBdr}_{M}$ iff $H=\operatorname{Outcm}_{M \cap h}(h)$ for an $h$ passing across $M$. Furthermore we have the following by definition and NBB:

Fact 3.1. Let $h$ pass across $M^{+}$and $h^{\prime} \in \operatorname{Outcm}_{M^{+} \cap h}(h)$. Then $h^{\prime}$ passes across $M^{+}, M^{+} \cap h=M^{+} \cap h^{\prime}$ and $\operatorname{Outcm}_{M^{+} \cap h}(h)=$ Outcm $_{M^{+} \cap h^{\prime}}\left(h^{\prime}\right)$. Consequently, for each outcome $H, H \in O u t c m B d r_{M}$ iff for each $h \in H, h$ passes across $M^{+}$and $H=$ Outcm $_{M^{+} \cap h}(h)$.

The next fact is a direct consequence of Facts 2.4, 2.5 and 3.1.

Fact 3.2. Let $\langle T,<$, Agent, Choice $\rangle$ be any stit frame, let $H \in O$ OutcmBdr $r_{M}$ with $M$ to be any field, and let $\mathcal{G}$ be any group. For each $x \in M$ and each $K \in$ Choice $_{\mathcal{G}}^{x}$, either $H \subseteq K$ or $H \cap K=\varnothing$.

The following propositions show some facts concerning fields and outcomes bordering them. The first states that no outcome bordering a field is compatible with another such outcome.

Proposition 3.3. Let $M$ be any field, and let $H, H^{\prime} \in O u t c m B d r_{M}$. Then $H \neq H^{\prime}$ only if $H \cap H^{\prime}=\varnothing$. Consequently, for all $U, U^{\prime} \subseteq O u t c m B d r_{M}, \bigcup U=\bigcup U^{\prime}$ iff $U=U^{\prime}$.

Proof. By definition, there are histories $h$ and $h^{\prime}$ passing across $M^{+}$such that $H=$ Outcm $_{c}(h)$ and $H^{\prime}=$ Outcm $_{c^{\prime}}\left(h^{\prime}\right)$ where $c=M^{+} \cap h$ and $c^{\prime}=M^{+} \cap h^{\prime}$. By Proposition 2.2, either $H \subseteq H^{\prime}$ or $H^{\prime} \subseteq H$ or $H \cap H^{\prime}=\varnothing$. If $H \subseteq H^{\prime}, h \in$ Outcm $_{c^{\prime}}\left(h^{\prime}\right)$, and then $H=H^{\prime}$ by Fact 3.1. Similarly, $H^{\prime} \subseteq H$ only if $H=H^{\prime}$. Hence $H \neq H^{\prime}$ only if $H \cap H^{\prime}=\varnothing$.

Proposition 3.4. Let $M$ be any properly covered field. Then,

(i) for each history $h, h \in H_{\langle M\rangle}$ iff $h$ passes across $M^{+}$;

(ii) for each $h \in H_{\langle M\rangle}$, there is an $H \in$ OutcmBdr $_{M}$ such that $h \in H$;

(iii) for each outcome $H, H \in \operatorname{OutcmBd}_{M}$ iff $H=\operatorname{Outcm}_{M^{+} \cap h}(h)$ for an $h \in H_{\langle M\rangle}$;

(iv) $H_{\langle M\rangle}=\bigcup$ OutcmBdr $r_{M}$ and $O u t c m B d r_{M}=O u t c m B d r_{M^{+}}$.

Proof. (i) Let $h \in H_{\langle M\rangle}$, i.e., $h \in H_{x}$ for an $x \in M$. Assume that $i$ properly covers $M$. Then $i$ intersects $h$ and $x<m_{i, h}$. If $m_{i, h} \leqslant z$ for a $z \in M^{+}, m_{i, h} \leqslant z^{\prime}$ for a $z^{\prime} \in M$, and then by definition, $z^{\prime}<u$ for a $u \in i$, and hence $m_{i, h}<u$, contrary to our assumption that $i$ is an anti-chain. It follows that $M^{+} \cap h<m_{i, h}$, and thus $h$ passes across $M^{+}$. (ii) For each $h \in H_{\langle M\rangle}, h$ passes across $M^{+}$by (i), and then $h \in$ Outcm $_{M^{+} \cap h}(h) \in$ OutcmBdr $_{M}$ by definition. (iii) follows from (i) by definition, and (iv) follows from (ii), (iii), and a simple fact that $M^{+}=M^{++}$.

Proposition 3.4 (ii) and the following establish that for each field $M, M$ is properly covered iff no matter which history we go along through $M$, we always go into an outcome at the "border" of $M$. From now on, we use "AC" to mark a proposition or a fact to indicate the dependence of our proof (or a routine proof) on the Axiom of Choice. 
Proposition 3.5. (AC). Let $M$ be any field such that for each $h \in H_{\langle M\rangle}$, there is an $H \in O u t c m B d r_{M}$ such that $h \in H$. Then $M$ is properly covered.

Proof. For each $H \in O u t c m B d r_{M}$, we know that there is an anti-chain $i_{H}$ such that $i_{H} \cap M=\varnothing$ and $i_{H}$ intersects all and only $h \in H$. Letting $i$ be the union of all $i_{H}$ with $H \in O u t c m B d r_{M}$, we know by Proposition 3.3 that $i$ is an anti-chain. It is then routine to verify that $i$ properly covers $M$.

Let $M$ be any properly covered field. By Propositions 3.3-3.4, we know that each history $h \in H_{\langle M\rangle}$ is contained in a unique $M$-bordering outcome. Thus we will use, for each $h \in H_{\langle M\rangle}$, OutcmBdr $r_{M}(h)$ for the unique $M$-bordering outcome to which $h$ belongs.

For each point $m, O u t c m B d r_{\{m\}}$ is obviously the set of all outcomes at $m$, i.e., OutcmBdr $r_{\{m\}}=$ Outcm $_{m}$. It is worth noting, however, that for a chain $c$ of points, $O u t c m B d r_{c}$ is not in general the same as $O u t c c_{c}$, and that for a field $M, O u t c m B d r_{M}$ is not in general the same as $\bigcup\left\{\right.$ Outcm $_{c}: c$ is a maximal chain in $\left.M^{+}\right\}$. For example, suppose that $h, h^{\prime} \in H_{x}$ and $\operatorname{Outcm}_{x}(h) \neq$ Outcm $_{x}\left(h^{\prime}\right)$ ( $h$ and $h^{\prime}$ share no point after $x)$. Let $M=\{x, y\}$ with $x<y \in h^{\prime}$. Then we can easily verify that $\operatorname{Outcm}_{x}(h)$ is $M$-bordering, although not an outcome at the chain $\{x, y\}$.

\section{Strategies and Their Admitted Future Outcomes}

The semantic account for ought sentences developed in Horty (2001) emphasizes a dominance relation between choices at a single moment, for the same agent or group. Despite its merits, the account has two limitations. On the one hand, what one ought to achieve is often not what she can do in a single choice or action, but in a series of choices or actions. On the other hand, we may take a current choice to dominate another not because the immediate outcomes ensured by the former have higher value than those ensured by the latter. It may be because, when we look further into the future possibilities, the former opens a series of actions leading to future outcomes that have higher values than those to which the latter may lead us. This is what brought Horty to his theory in Horty (2001) of strategic ought with a single agent.

There are nevertheless some problems when Horty approaches his notions of strategic acts and strategic oughts, one of which is related to the notion of independence concerning choices for different agents at different moments. The problems are not really in the theories of strategies developed by Belnap and Horty, but in their applications or relations to other theories. We may then proceed safely to expand their theories of strategies, and discuss the problems in some other place.

This section and the following two expand Belnap's theory of strategies to the extent that we can talk about what different groups may do in the same strategy field. In doing so, we restrict ourselves to "primary strategies", as Belnap calls them, or "irredundant strategies", as Horty calls them. Notions and most terms are inherited 
directly from Belnap (1996b) and Belnap et al. (2001). Throughout the rest of this paper, we fix $\langle T,<$, Agent, Choice $\rangle$ to be a stit frame, relative to which all upcoming discussions are to be understood.

A strategy for a group $\mathcal{G}$ in a field $M$ is a function $s$ such that $\operatorname{dom}(s) \subseteq M$, where $\operatorname{dom}(s)$ is the domain of $s$, and $s(x) \in$ Choice $_{\mathcal{G}}^{x}$ for each $x \in \operatorname{dom}(s)$. A strategy for $\mathcal{G}$ is a strategy for $\mathcal{G}$ in a field, and a strategy (in a field) is a strategy for a group (in the field). A strategy for an individual agent $\alpha$ (in a field) is a strategy for $\{\alpha\}$ (in the field). ${ }^{12}$ We have assumed that a field is always nonempty, and now we further assume that so is every strategy in every field (with functions to be identified with sets of ordered pairs). Here are some basic notions concerning strategies.

Definition 4.1. Let $s$ be any strategy, $h$ any history, $m$ any moment, $H$ any outcome, and $M$ any field. Then

(i) $s$ admits $h$ iff $h \in s(x)$ for each $x \in \operatorname{dom}(s) \cap h,^{13}$

(ii) $\operatorname{adh}(s)=\left\{h^{\prime}: s\right.$ admits $\left.h^{\prime}\right\}$,

(iii) $s$ admits $m$ iff $m \in h$ for an $h \in \operatorname{adh}(s)$,

(iv) $\operatorname{adm}(s)=\{x: s$ admits $x\}$,

(v) $s$ admits $H$ iff $H \subseteq$ adh(s),

(vi) $\operatorname{ado}_{M}(s)=\left\{H^{\prime} \in\right.$ OutcmBdr $_{M}: s$ admits $\left.H^{\prime}\right\}$.

Concerning the new notion of admitted outcomes bordering a field $M$, it is easy to verify by definition that $\operatorname{Uado}_{M}(s) \subseteq \operatorname{adh}(s)$ for each strategy $s$ in $M$, and hence the following fact holds:

Fact 4.2. Let $M$ be any field in which $s$ is a strategy for a group $\mathcal{G}$. Then for each $H \in \operatorname{OutcmBdr}_{M}, H \subseteq \bigcup a d o_{M}(s)$ iff $H \in \operatorname{ado}_{M}(s)$.

Definition 4.3. Let $s$ be any strategy for $\mathcal{G}$ in a field $M$. Then

(i) $s$ is primary iff $\operatorname{dom}(s) \subseteq \operatorname{adm}(s)$;

(ii) $s$ is secondary iff it is not primary;

(iii) $s$ is backward closed in $M$ iff for all $x, y \in M, x \in \operatorname{dom}(s)$ and $y<x$ only if $y \in \operatorname{dom}(s)$;

(iv) $s$ is simple in $M$ iff it is primary and backward closed in $M$.

For each group $\mathcal{G}$, we use $P$-Strategy $y_{\mathcal{G}}^{M}\left(S\right.$-Strategy $\left.y_{\mathcal{G}}^{M}\right)$ for the set of all primary (simple) strategies for $\mathcal{G}$ in $M$. The realm of primary strategies is our focus in this paper. ${ }^{14}$ Note that for each $s \in P$-Strategy ${ }_{\mathcal{G}}^{M}$ and each $x \in \operatorname{dom}(s), s(x) \cap \operatorname{adh}(s) \neq \varnothing$ by definition, and hence $a d h(s) \cap H_{\langle M\rangle}$ is never empty. Furthermore, the following fact can easily be verified.

\footnotetext{
${ }^{12}$ Belnap calls such a function a consistent and strict strategy for $\alpha$ in $M$ in Belnap (1996b) and Belnap et al. (2001), while Horty calls it a strategy for $\alpha$ in $M$ in Horty (2001), though the field $M$ in the latter needs to have a starting point up to which $M$ is backward closed.

${ }^{13}$ In Horty (2001), for $s$ to admit $h$, it is further required that $h \cap \operatorname{dom}(s) \neq \varnothing$.

${ }^{14}$ Secondary strategies are important for a study of conditional ought with respect to future outcomes, though they are not in the scope of our current work. For a brief discussion of secondary strategies, see Belnap (1996b) or Belnap et al. (2001).
} 
Fact 4.4. (AC). For each primary strategy $s$, each nonempty chain in $\operatorname{dom}(s)$ can be extended to a history that $s$ admits. ${ }^{15}$

The following propositions establish some simple connections between admitted histories and admitted outcomes, the first of which states that a strategy in $M$ admits an $M$-bordering outcome if it admits a member of it.

Proposition 4.5. Let $s$ be a strategy for $\mathcal{G}$ in a field $M$. Then for each $H \in$ $\operatorname{OutcmBdr}_{M}, H \in \operatorname{ado}_{M}(s)$ iff $H \cap \operatorname{adh}(s) \neq \varnothing$.

Proof. Letting $H \in O$ OutcmBdr $r_{M}$, we show that $h \in H \cap \operatorname{adh}(s)$ only if $H \subseteq \operatorname{adh}(s)$. Suppose that $h \in H \cap \operatorname{adh}(s)$. Let $c=M^{+} \cap h$. Then $c \neq \varnothing$ and $H=\operatorname{Outcm}_{c}(h)$ by Fact 3.1. Consider any $h^{\prime} \in H$ and any $x \in \operatorname{dom}(s) \cap h^{\prime}$. We know that $x \in M \cap h^{\prime} \subseteq$ $c \subseteq h \cap h^{\prime}$, and thus by Facts 2.4-2.5, Outcm ${ }_{c}(h) \subseteq$ Outcm $_{x}(h) \subseteq$ Choice $_{\mathcal{G}}^{x}(h)$. Then Outcm $_{c}(h) \subseteq s(x)=$ Choice $_{\mathcal{G}}^{x}(h)$ since $h \in \operatorname{adh}(s)$, and hence $h^{\prime} \in s(x)$ since $h^{\prime} \in \operatorname{Outcm}_{c}(h)$. It follows that $h^{\prime} \in s(x)$ for every $x \in \operatorname{dom}(s) \cap h^{\prime}$, and hence $h^{\prime} \in \operatorname{adh}(s)$.

The following proposition is useful when we extend our results concerning admitted histories passing through a field to similar results concerning admitted outcomes bordering the field.

Proposition 4.6. Let $M$ be any properly covered field, and let $s$ be any strategy in $M$. Then $\bigcup_{\operatorname{ado}}(s)=\operatorname{adh}(s) \cap H_{\langle M\rangle}$.

Proof. By definition, $\operatorname{Jado}_{M}(s) \subseteq \operatorname{adh}(s)$ and $\operatorname{Uado}_{M}(s) \subseteq \cup \operatorname{UutcmBd}_{M}$, and hence $\bigcup a d o_{M}(s) \subseteq a d h(s) \cap H_{\langle M\rangle}$ by Proposition 3.4 (iv). Consider any $h \in \operatorname{adh}(s) \cap H_{\langle M\rangle}$. By Proposition 3.4 (ii), $h \in H$ for an $H \in$ OutcmBdr $_{M}$, and then, since $h \in \operatorname{adh}(s)$, Proposition 4.5 implies that $H \in \operatorname{ado}_{M}(s)$, and hence $h \in \bigcup_{\operatorname{ado}}(s)$. It follows that $\operatorname{Uado}_{M}(s)=\operatorname{adh}(s) \cap H_{\langle M\rangle}$.

\section{Pre-Simple Strategies and Complete Strategies}

Here we present a brief discussion on pre-simple strategies and complete primary strategies. The proofs of propositions in this section follow closely those in Chap. 13 of Belnap et al. (2001), except that we expand various notions there concerning individual strategies to those concerning group strategies. Readers familiar with the materials in Chap. 13 of Belnap et al. (2001) may skip this section.

Recall that when $y<x$, we use Choice $_{\mathcal{G}}^{y}\left(H_{x}\right)$ for the unique $K \in$ Choice $_{\mathcal{G}}^{y}$ such that $H_{x} \subseteq K$ (see Fact 1.1). A strategy $s$ in a field $M$ is pre-simple in $M$ iff $s$ is primary, and for all $x, y \in \operatorname{dom}(s)$ and $z \in M, z<x$ and $z<y$ only if Choice $_{\mathcal{G}}^{z}\left(H_{x}\right)=$ Choice $_{\mathcal{G}}^{z}\left(H_{y}\right)$.

For all strategies $s$ and $s^{\prime}$ for a group $\mathcal{G}, s^{\prime}$ is an extension of $s$ (or $s^{\prime}$ extends $s$ ) iff $s \subseteq s^{\prime}$, where we identify functions as sets of ordered pairs. Note that when speaking

\footnotetext{
${ }^{15}$ It is also easy to verify that this does not hold for secondary strategies.
} 
of an extension $s^{\prime}$ of a strategy $s$, we always presuppose that $s$ and $s^{\prime}$ are strategies for the same group. Note also that if $s^{\prime}$ extends $s$, then by definition, $\operatorname{adh}\left(s^{\prime}\right) \subseteq \operatorname{adh}(s)$. A simple (primary) extension of a strategy $s$ in $M$ is an extension of $s$ that is itself simple (primary) in $M$. A primary strategy in $M$ may have no simple extension at all in $M$, but each pre-simple strategy in $M$ does have such an extension.

Proposition 5.1. (AC). $s$ is pre-simple for $\mathcal{G}$ in $M$ iff it can be extended to a simple strategy for $\mathcal{G}$ in $M$.

Proof. Suppose that $s$ is pre-simple for $\mathcal{G}$ in $M$. Let $D=\{y \in M-\operatorname{dom}(s): \exists x \in$ $\operatorname{dom}(s)(y<x)\}$. Consider any $y \in D$. Because $s$ is pre-simple in $M$, there is a unique $K_{y} \in$ Choice $_{\mathcal{G}}^{y}$ such that $H_{x} \subseteq K_{y}$ for each $x \in \operatorname{dom}(s)$ with $y<x$. Let $s^{\prime}=s \cup\left\{\left\langle y, K_{y}\right\rangle: y \in D\right\}$. It is easy to verify that $s^{\prime}$ is a backward closed extension of $s$ in $M$, and then $a d h\left(s^{\prime}\right) \subseteq a d h(s)$ by definition. To show that $s^{\prime}$ is primary, consider any $x \in \operatorname{dom}\left(s^{\prime}\right)=\operatorname{dom}(s) \cup D$. Then there is a $u \in \operatorname{dom}(s)$ such that $x \leqslant u$, and then, letting $c$ be a maximal chain in $\operatorname{dom}(s)$ containing $u$, we know by Fact 4.4 that $c=h \cap \operatorname{dom}(s)$ for an $h \in \operatorname{adh}(s)$. For each $y \in h \cap \operatorname{dom}\left(s^{\prime}\right)$, if $y \in \operatorname{dom}(s), h \in s(y)=s^{\prime}(y)$ since $h \in \operatorname{adh}(s)$; and if $y \in D, y<z$ for a $z \in c$ by the maximality of $c$ in $\operatorname{dom}(s)$, and then $h \in s(z) \subseteq s^{\prime}(y)$ by definition of $s^{\prime}$. It follows that $h \in \operatorname{adh}\left(s^{\prime}\right)$, and then, since $x \leqslant u \in h, x \in \operatorname{adm}\left(s^{\prime}\right)$. Hence $s^{\prime}$ is primary.

Suppose that $s$ is not pre-simple in $M$. If $s$ is secondary, there is an $x \in \operatorname{dom}(s)$ such that $H_{x} \cap \operatorname{adh}(s)=\varnothing$, and then for each extension $s^{\prime}$ of $s, \operatorname{adh}\left(s^{\prime}\right) \subseteq \operatorname{adh}(s)$, and thus $H_{x} \cap a d h\left(s^{\prime}\right)=\varnothing$, and hence $s^{\prime}$ is secondary. Assume that $s$ is primary. Then for some $x, y \in \operatorname{dom}(s)$ and $z \in M, z<x$ and $z<y$, and Choice $_{\mathcal{G}}^{z}\left(H_{x}\right) \neq$ Choice $_{\mathcal{G}}^{z}\left(H_{y}\right)$. Consider any backward closed extension $s^{\prime}$ of $s$ in $M$. If $s^{\prime}(z) \neq \operatorname{Choice}_{\mathcal{G}}^{z}\left(H_{x}\right)$, $H_{x} \cap \operatorname{adh}\left(s^{\prime}\right)=\varnothing$, and then $x \notin \operatorname{adm}\left(s^{\prime}\right)$; and similarly, if $s^{\prime}(z) \neq \operatorname{Choice}_{\mathcal{G}}^{z}\left(H_{y}\right)$, $y \notin \operatorname{adm}\left(s^{\prime}\right)$. Since either $s^{\prime}(z) \neq \operatorname{Choice}_{\mathcal{G}}^{z}\left(H_{x}\right)$ or $s^{\prime}(z) \neq \operatorname{Choice}_{\mathcal{G}}^{z}\left(H_{y}\right)$, either $x \notin \operatorname{adm}\left(s^{\prime}\right)$ or $y \notin \operatorname{adm}\left(s^{\prime}\right)$, which makes $s^{\prime}$ secondary.

A complete strategy in a field is a strategy that is defined everywhere in the field along its admitted histories.

Definition 5.2. Let $s$ be any strategy for $\mathcal{G}$ in a field $M$. Then

(i) $s$ is complete along a history $h$ in $M$ iff $M \cap h \subseteq \operatorname{dom}(s)$;

(ii) $s$ completely admits $h$ in $M$ iff $s$ admits $h$ and is complete along $h$ in $M$;

(iii) $s$ is complete in $M$ iff $s$ is complete along every $h \in \operatorname{adh}(s)$ in $M$.

The fact below is a direct consequence of our definitions.

Fact 5.3. Let $s$ and $s^{\prime}$ be any strategies for $\mathcal{G}$ in $M$ such that $s^{\prime}$ extends $s$. Then the following hold:

(i) $s$ is complete along $h$ in $M$ only if $s^{\prime}$ is;

(ii) $s$ completely admits $h$ in $M$ only if $s^{\prime}$ does. 
We will use $C P$-Strategy $y_{\mathcal{G}}^{M}$ for the set of all complete primary strategies for $\mathcal{G}$ in $M$. It is obvious that $C P$-Strategy ${ }_{\mathcal{G}}^{M} \subseteq P$-Strategy $y_{\mathcal{G}}^{M}$. The following facts prove useful:

Fact 5.4. Let $M$ be any field, and let $\mathcal{F}$ and $\mathcal{G}$ be any groups. Then

(i) $C P$-Strategy $y_{\mathcal{G}}^{M} \subseteq S$-Strategy $\mathcal{G}_{\mathcal{G}}^{M}$;

(ii) for each $s \in C P$-Strategy $y_{\mathcal{G}}^{M}$ and each $h \in H_{\langle M\rangle}, h \cap \operatorname{dom}(s) \neq \varnothing$;

(iii) for each $s \in C P$-Strategy $y_{\mathcal{F}}^{M}$ and each $s^{\prime} \in C P$-Strategy $y_{\mathcal{G}}^{M}, \operatorname{dom}(s) \cap \operatorname{dom}\left(s^{\prime}\right) \neq \varnothing$.

Proof. (i) Let $s \in C P$-Strategy $\mathcal{G}_{\mathcal{G}}^{M}$. For each $x \in \operatorname{dom}(s)$, since $s$ is primary, $h \in s(x)$ for an $h \in a d h(s)$, and then, since $s$ is complete along $h, M \cap\{y: y<x\} \subseteq$ $M \cap h \subseteq \operatorname{dom}(s)$. It follows that $s$ is backward closed, and then $s \in S$-Strategy $y_{\mathcal{G}}^{M}$. (ii) Let $s \in C P$-Strategy $y_{\mathcal{G}}^{M}$ and $h \in H_{\langle M\rangle}$. If $h \cap \operatorname{dom}(s)=\varnothing$, then trivially $h \in \operatorname{adh}(s)$, and then $h \cap M \subseteq \operatorname{dom}(s)$ since $s$ is complete along $h$ in $M$, and hence $h \cap M \subseteq$ $h \cap \operatorname{dom}(s)=\varnothing$, contrary to that $h \in H_{\langle M\rangle}$. (iii) follows from (i) and (ii).

For each strategy $s$ for $\mathcal{G}$ in $M$, and for each $h \in \operatorname{adh}(s)$, let $s_{h}$ be a function on $\operatorname{dom}(s) \cup(h \cap M)$ such that $s_{h}(x)=s(x)$ for each $x \in \operatorname{dom}(s)$, and $s_{h}(x)=$ Choice $_{\mathcal{G}}^{x}(h)$ for each $x \in(h \cap M)-\operatorname{dom}(s)$. Such $s_{h}$ is obviously unique, and is a strategy for $\mathcal{G}$ in $M$ which extends $s$, and we say that $s^{\prime}$ extends $s$ (completely) along $h$ in $M$ iff $s^{\prime}=s_{h}$.

Proposition 5.5. Let $s$ be a strategy for $\mathcal{G}$ in $M$, let $h \in \operatorname{adh}(s)$, and let $s^{\prime}$ extend $s$ along $h$ in $M$. Then the following hold:

(i) $s^{\prime}$ completely admits $h$ in $M$;

(ii) $s$ is simple in $M$ only if $s^{\prime}$ is.

Proof. (i) It is clear by definition that $s^{\prime}$ is complete along $h$ in $M$, and $h \cap \operatorname{dom}\left(s^{\prime}\right)=$ $D \cup(\operatorname{dom}(s) \cap h)$, where $D=(h \cap M)-\operatorname{dom}(s)$. Also by definition, $h \in$ Choice $_{\mathcal{G}}^{x}(h)=s^{\prime}(x)$ for each $x \in D$, and, since $h \in \operatorname{adh}(s), h \in s(y)=s^{\prime}(y)$ for each $y \in \operatorname{dom}(s) \cap h$. It follows that $h \in \operatorname{adh}\left(s^{\prime}\right)$.

(ii) Let $s$ be simple in $M$. Then $s^{\prime}$ is evidently backward closed in $M$. To show that $s^{\prime}$ is primary, consider any $x \in \operatorname{dom}\left(s^{\prime}\right)$. If $x \in h, x \in \operatorname{adm}\left(s^{\prime}\right)$ since $h \in \operatorname{adh}\left(s^{\prime}\right)$ by (i). Suppose that $x \in \operatorname{dom}\left(s^{\prime}\right)-h$. Then $x \in \operatorname{dom}(s)-h$. Since $s$ is primary, $x \in h^{\prime}$ for an $h^{\prime} \in \operatorname{adh}(s)$. Now for each $y \in \operatorname{dom}\left(s^{\prime}\right) \cap h^{\prime}$, if $y<x, y \in \operatorname{dom}(s)$ since $s$ is backward closed in $M$, and if $x \leqslant y, y \notin h$ since $x \notin h$, and hence $y \in \operatorname{dom}(s)$. It follows that for each $y \in \operatorname{dom}\left(s^{\prime}\right) \cap h^{\prime}, y \in \operatorname{dom}(s)$ and then $s^{\prime}(y)=s(y)$, which implies that $h^{\prime} \in s^{\prime}(y)$ since $h^{\prime} \in \operatorname{adh}(s)$. Hence $h^{\prime} \in \operatorname{adh}\left(s^{\prime}\right)$, and then $x \in \operatorname{adm}\left(s^{\prime}\right)$

A complete primary extension of a strategy $s$ for $\mathcal{G}$ in $M$ is an $s^{\prime} \in C P$-Strategy $y_{\mathcal{G}}^{M}$ such that $s \subseteq s^{\prime}$. We show below that each pre-simple strategy has a complete primary extension.

Proposition 5.6. (AC). Let $S$ be a nonempty $\subseteq$-chain of simple strategies for $\mathcal{G}$ in $M$. Then $\bigcup S$ is a simple strategy for $\mathcal{G}$ in $M$ that extends all $s^{\prime} \in S$. 
Proof. Let $s=\bigcup S$. It is easy to see that $s$ is a strategy for $\mathcal{G}$ in $M$ extending all $s^{\prime} \in S$, and is backward closed in $M$ since each $s^{\prime} \in S$ is. It then suffices to let $x \in \operatorname{dom}(s)$ and show that $x \in h$ for an $h \in \operatorname{adh}(s)$. Let $c$ be a maximal chain in $\operatorname{dom}(s)$ containing $x$. Case 1, $c$ contains a largest member $u$. Then $u \in \operatorname{dom}\left(s^{\prime}\right)$ for an $s^{\prime} \in S$, and $s^{\prime}(y)=s(y)$ for each $y \in \operatorname{dom}(s)$ with $y \leqslant u$. Since $s^{\prime}$ is primary, $u \in h$ for an $h \in \operatorname{adh}\left(s^{\prime}\right)$, and then, because $\operatorname{dom}(s) \cap h=\operatorname{dom}\left(s^{\prime}\right) \cap h$, it follows that $h \in s^{\prime}(y)=s(y)$ for each $y \in \operatorname{dom}(s) \cap h$, i.e., $h \in \operatorname{adh}(s)$. Case 2, $c$ has no largest member. Let $h$ be any history including $c$. Consider any $y \in \operatorname{dom}(s) \cap h$. Then there is a $z \in \operatorname{dom}(s) \cap h$ and an $s^{\prime \prime} \in S$ such that $y, z \in \operatorname{dom}\left(s^{\prime \prime}\right), y<z$ and $s(y)=s^{\prime \prime}(y)$. Since $s^{\prime \prime}$ is primary, $z \in h^{\prime}$ for some $h^{\prime} \in \operatorname{adh}\left(s^{\prime \prime}\right)$, and then, since $h, h^{\prime} \in H_{z}$ and $y<z, h \in s^{\prime \prime}(y)=s(y)$ by NC (see Sect. 1). It follows that $h \in \operatorname{adh}(s)$.

Applying Zorn's lemma, Fact 5.3 and Propositions 5.5-5.6 and 5.1, one can routinely establish the following.

Proposition 5.7. (AC). For each $s \in S$-Strategy ${ }_{\mathcal{G}}^{M}$ and each $h \in \operatorname{adh}(s), s \subseteq s^{\prime}$ and $h \in \operatorname{adh}\left(s^{\prime}\right)$ for an $s^{\prime} \in C P$-Strategy ${ }_{\mathcal{G}}^{M}$, and hence $\operatorname{adh}(s)=\bigcup_{s^{\prime \prime} \in S} a d h\left(s^{\prime \prime}\right)$ where $S=\left\{s^{\prime \prime} \in C P\right.$-Strategy $\left.y_{\mathcal{G}}^{M}: s \subseteq s^{\prime \prime}\right\}$. Consequently, each pre-simple strategy for $\mathcal{G}$ in $M$ has a complete primary extension in $M$.

\section{Group-Joining Meets}

Bringing in different agents and their strategies provides new perspectives to a study of strategies for different agents in the same fields. As a preparation for our discussions on distinguishability and independence, we deal with some technical notions in this section.

Consider two agents $\alpha$ and $\beta$, and their strategies $s_{\alpha}$ and $s_{\beta}$ in a field $M$ with $m \in D=\operatorname{dom}\left(s_{\alpha}\right) \cap \operatorname{dom}\left(s_{\beta}\right)$. Since $\alpha \neq \beta$, IA requires $s_{\alpha}(m) \cap s_{\beta}(m) \neq \varnothing$, and the same can be said about each point in $D$. Letting $s$ be a function on $D$ such that $s(x)=s_{\alpha}(x) \cap s_{\beta}(x)$ for each $x \in D$, we know that each $s(x)$ with $x \in D$ is a member of Choice $e_{\{\alpha, \beta\}}^{x}$, and hence $s$ is a strategy for $\{\alpha, \beta\}$ in $M$. This strategy is what we call the "group-joining meet" of $s_{\alpha}$ and $s_{\beta}$.

Definition 6.1. Let $s$ and $s^{\prime}$ be strategies in $M$ for $\mathcal{G}$ and $\mathcal{F}$ respectively such that $\mathcal{G} \cap \mathcal{F}=\varnothing$ and $\operatorname{dom}(s) \cap \operatorname{dom}\left(s^{\prime}\right) \neq \varnothing$. The group-joining meet of $s$ and $s^{\prime}$, written $s \sqcap s^{\prime}$, is the function $s^{*}$ on $\operatorname{dom}\left(s^{*}\right)=\operatorname{dom}(s) \cap \operatorname{dom}\left(s^{\prime}\right)$ such that $s^{*}(x)=s(x) \cap s^{\prime}(x)$ for every $x \in \operatorname{dom}\left(s^{*}\right)$.

It is easy to see that $s \sqcap s^{\prime}=s^{\prime} \sqcap s$ when both are defined. When $\mathcal{G}$ and $\mathcal{F}$ are disjoint while $\operatorname{dom}(s)$ and $\operatorname{dom}\left(s^{\prime}\right)$ are not, we know that for each $x \in \operatorname{dom}\left(s \sqcap s^{\prime}\right)$, $\left(s \sqcap s^{\prime}\right)(x)$ is not only nonempty, but also identical to a member of Choice $\mathcal{G}_{\mathcal{G}}^{x}$. It then follows that $s \sqcap s^{\prime}$ is a strategy for $\mathcal{G} \cup \mathcal{F}$ in $M$.

Before showing some facts concerning strategies and their group-joining meets, we need to show that for all primary strategies $s$ and $s^{\prime}$ for $\mathcal{F}$ and $\mathcal{G}$ respectively, if 
$\mathcal{F}$ and $\mathcal{G}$ are disjoint but $\operatorname{dom}(s)$ and $\operatorname{dom}\left(s^{\prime}\right)$ are not, then $\operatorname{adh}(s) \cap \operatorname{adh}\left(s^{\prime}\right) \neq \varnothing$. To that end, we use the following auxiliary notion. Let $s$ and $s^{\prime}$ be strategies for $\mathcal{G}$ in $M$, and let $h \in H_{\langle M\rangle}$ and $c \subseteq h \cap M$. $s^{\prime}$ extends $s$ in $M$ along $c$ w.r.t. $h$ if $s^{\prime}$ extends $s$ such that $\operatorname{dom}\left(s^{\prime}\right)=\operatorname{dom}(s) \cup c$ and $s^{\prime}(x)=\operatorname{Choice}_{\mathcal{G}}^{x}(h)$ for each $x \in h \cap(c-\operatorname{dom}(s))$. Note that if $s^{\prime}$ is an extension of $s$ in $M$ along $c$ w.r.t. $h$, then such $s^{\prime}$ is unique, and $h \in \operatorname{adh}(s)$ only if $h \in \operatorname{adh}\left(s^{\prime}\right)$.

Proposition 6.2. (AC). Let $s_{\mathcal{F}}$ and $s_{\mathcal{G}}$ be primary strategies for $\mathcal{F}$ and $\mathcal{G}$ in $M$ respectively, where $\mathcal{F} \cap \mathcal{G}=\varnothing$, and let $m \in \operatorname{dom}\left(s_{\mathcal{F}}\right) \cap \operatorname{dom}\left(s_{\mathcal{G}}\right) .{ }^{16}$ Then $\operatorname{adh}\left(s_{\mathcal{F}}\right) \cap$ $\operatorname{adh}\left(s_{\mathcal{G}}\right) \cap H_{m} \neq \varnothing$, and $a d o_{M}\left(s_{\mathcal{F}}\right) \cap a d o_{M}\left(s_{\mathcal{G}}\right) \neq \varnothing$ if $M$ is properly covered.

Proof. Let $D_{\mathcal{F}}=\left\{x \in M: \exists y\left(x \leqslant y \in \operatorname{dom}\left(s_{\mathcal{F}}\right)\right)\right\}$ and $D_{\mathcal{G}}=\{x \in M: \exists y(x \leqslant$ $\left.\left.y \in \operatorname{dom}\left(s_{\mathcal{G}}\right)\right)\right\}$. By hypothesis, $m \in D_{\mathcal{F}} \cap D_{\mathcal{G}}$. Let $c$ be a maximal chain in $D_{\mathcal{F}} \cap D_{\mathcal{G}}$ containing $m$. It is easy to verify that $H_{[c]} \subseteq H_{m}$ and

$$
\text { for each } h \in H_{[c]}, h \cap \operatorname{dom}\left(s_{\mathcal{F}}\right) \subseteq c \text { or } h \cap \operatorname{dom}\left(s_{\mathcal{G}}\right) \subseteq c .
$$

Let $d_{\mathcal{F}}$ and $d_{\mathcal{G}}$ be any maximal chains, extending $c$, in $D_{\mathcal{F}}$ and $D_{\mathcal{G}}$ respectively. It is easy to see that $d_{\mathcal{F}} \cap \operatorname{dom}\left(s_{\mathcal{F}}\right)$ is a maximal chain in $\operatorname{dom}\left(s_{\mathcal{F}}\right)$ that is co-final with $d_{\mathcal{F}}$, and therefore can by Fact 4.4 be extended to an $h_{\mathcal{F}} \in \operatorname{adh}\left(s_{\mathcal{F}}\right)$ such that $c \subseteq d_{\mathcal{F}} \subseteq h_{\mathcal{F}}$. Similarly, $c \subseteq d_{\mathcal{G}} \subseteq h_{\mathcal{G}}$ for an $h_{\mathcal{G}} \in \operatorname{adh}\left(s_{\mathcal{G}}\right)$. Let $s_{\mathcal{F}}^{\prime}$ be the extension of $s_{\mathcal{F}}$ in $M$ along $c$ w.r.t. $h_{\mathcal{F}}$, and let $s_{\mathcal{G}}^{\prime}$ be the extension of $s_{\mathcal{G}}$ in $M$ along $c$ w.r.t. $h_{\mathcal{G}}$. By our note above,

$$
h_{\mathcal{F}} \in \operatorname{adh}\left(s_{\mathcal{F}}^{\prime}\right) \subseteq \operatorname{adh}\left(s_{\mathcal{F}}\right) \text { and } h_{\mathcal{G}} \in \operatorname{adh}\left(s_{\mathcal{G}}^{\prime}\right) \subseteq \operatorname{adh}\left(s_{\mathcal{G}}\right)
$$

By Propositions 3.4 (ii) and 4.5, it suffices to show that

$$
\operatorname{adh}\left(s_{\mathcal{F}}\right) \cap \operatorname{adh}\left(s_{\mathcal{G}}\right) \cap H_{[c]} \neq \varnothing .
$$

Case 1 , there is no last point in $c$. By (3), $h_{\mathcal{F}}, h_{\mathcal{G}} \in s_{\mathcal{F}}^{\prime}(x) \cap s_{\mathcal{G}}^{\prime}(x)$ for each $x \in c$. It is then easy to see by our case assumption and NC that

$$
\text { for each } x \in c, H_{[c]} \subseteq s_{\mathcal{F}}^{\prime}(x) \cap s_{\mathcal{G}}^{\prime}(x)
$$

Assume that $h_{\mathcal{F}} \notin a d h\left(s_{\mathcal{G}}^{\prime}\right)$, or by (3) there is nothing more to show. Then there is a $y \in h_{\mathcal{F}} \cap \operatorname{dom}\left(s_{\mathcal{G}}^{\prime}\right)$ such that $h_{\mathcal{F}} \notin s_{\mathcal{G}}^{\prime}(y)$, and then by (5), $c<y \in h_{\mathcal{F}} \cap \operatorname{dom}\left(s_{\mathcal{G}}\right)$, and hence by (2), $h \cap \operatorname{dom}\left(s_{\mathcal{F}}\right) \subseteq c$ for each $h \in H_{y}$. It follows from (5) that $H_{y} \subseteq \operatorname{adh}\left(s_{\mathcal{F}}^{\prime}\right)$. Since $s_{\mathcal{G}}$ is primary, there is an $h^{\prime} \in H_{y} \cap \operatorname{adh}\left(s_{\mathcal{G}}\right)$, and hence (4) holds.

Case 2, there is a last point $z$ in $c$. Let $H=s_{\mathcal{F}}^{\prime}(z) \cap s_{\mathcal{G}}^{\prime}(z)$ and $X=(\bigcup H) \cap\{y$ : $z<y$. Because $\mathcal{F} \cap \mathcal{G}=\varnothing, H \neq \varnothing$ by IA. We claim that

\footnotetext{
16 The condition that $m \in \operatorname{dom}\left(s_{\mathcal{G}}\right) \cap \operatorname{dom}\left(s_{\mathcal{F}}\right)$ can be weakened to that $m \in M$ such that $m \leqslant x$ and $m \leqslant y$ for an $x \in \operatorname{dom}\left(s_{\mathcal{G}}\right)$ and a $y \in \operatorname{dom}\left(s_{\mathcal{F}}\right)$.
} 


$$
\text { for each } x \in c, H \subseteq s_{\mathcal{F}}^{\prime}(x) \cap s_{\mathcal{G}}^{\prime}(x)
$$

For each $x<z$ and $h \in H$, because $h, h_{\mathcal{F}}, h_{\mathcal{G}} \in H_{z}$, we know by NC and (3) that $h \in$ Choice $_{\mathcal{F}}^{x}\left(h_{\mathcal{F}}\right)=s_{\mathcal{F}}^{\prime}(x)$, and similarly, $h \in s_{\mathcal{G}}^{\prime}(x)$. Hence (6) holds. Subcase $2 \mathrm{~A}$, there is an $x \in \operatorname{dom}\left(s_{\mathcal{F}}^{\prime}\right) \cap X$. Then $c \leqslant z<x \in \operatorname{dom}\left(s_{\mathcal{F}}\right)$, and hence by (2), $h^{\prime} \cap \operatorname{dom}\left(s_{\mathcal{G}}\right) \subseteq c$ for each $h^{\prime} \in H_{x}$, and then $h^{\prime} \cap \operatorname{dom}\left(s_{\mathcal{G}}^{\prime}\right) \subseteq c$ for each $h^{\prime} \in H_{x}$ because $\operatorname{dom}\left(s_{\mathcal{G}}^{\prime}\right)-\operatorname{dom}\left(s_{\mathcal{G}}\right) \subseteq c$. It then follows from $H_{x} \subseteq H$ and (6) that $H_{x} \subseteq \operatorname{adh}\left(s_{\mathcal{G}}^{\prime}\right) \subseteq \operatorname{adh}\left(s_{\mathcal{G}}\right)$. Since $s_{\mathcal{F}}$ is primary, there is an $h \in H_{x} \cap \operatorname{adh}\left(s_{\mathcal{F}}\right)$, and then (4) holds. Subcase $2 \mathrm{~B}$, $\operatorname{dom}\left(s_{\mathcal{F}}^{\prime}\right) \cap X=\varnothing$. If there is a $u \in \operatorname{dom}\left(s_{\mathcal{G}}^{\prime}\right) \cap X$, an argument similar to that in subcase $2 \mathrm{~A}$ will show that (4) holds. If $\operatorname{dom}\left(s_{\mathcal{G}}^{\prime}\right) \cap X=\varnothing$, then (6) implies (4).

The following proposition provides a list of useful facts concerning strategies and their group-joining meets.

Proposition 6.3. Let $s$ and $s^{\prime}$ be strategies for $\mathcal{G}$ and $\mathcal{F}$ in $M$ respectively, where $\mathcal{G} \cap \mathcal{F}=\varnothing$ and $\operatorname{dom}(s) \cap \operatorname{dom}\left(s^{\prime}\right) \neq \varnothing$, and let $s^{*}=s \sqcap s^{\prime}$. Then

(i) $\operatorname{adh}(s) \cap \operatorname{adh}\left(s^{\prime}\right) \subseteq \operatorname{adh}\left(s^{*}\right)$;

(ii) $\operatorname{adm}(s) \cap \operatorname{adm}\left(s^{\prime}\right) \subseteq \operatorname{adm}\left(s^{*}\right)$ if both $s$ and $s^{\prime}$ are primary; (AC)

(iii) $\operatorname{adh}\left(s^{*}\right) \subseteq \operatorname{adh}(s)$ and $\operatorname{adm}\left(s^{*}\right) \subseteq \operatorname{adm}(s)$ if $s$ and $s^{\prime}$ are backward closed and $s^{\prime}$ is complete in $M$;

(iv) $\operatorname{adh}\left(s^{*}\right)=\operatorname{adh}(s) \cap a d h\left(s^{\prime}\right)$ if $s$ and $s^{\prime}$ are both backward closed and complete in $M$;

(v) $\operatorname{ado}_{M}\left(s^{*}\right)=\operatorname{ado}_{M}(s) \cap \operatorname{ado}_{M}\left(s^{\prime}\right)$ if $M$ is properly covered in which $s$ and $s^{\prime}$ are both backward closed and complete;

(vi) $\operatorname{adm}\left(s^{*}\right)=\operatorname{adm}(s) \cap \operatorname{adm}\left(s^{\prime}\right)$ if $s$ and $s^{\prime}$ are both primary and complete in $M$; (AC)

(vii) $s^{*}$ is backward closed in $M$ if both $s$ and $s^{\prime}$ are;

(viii) $s^{*}$ is primary (simple) in $M$ if both $s$ and $s^{\prime}$ are; (AC)

(ix) $s^{*}$ completely admits $h$ in $M$ if both $s$ and $s^{\prime}$ do;

(x) $s^{*}$ is backward closed and complete in $M$ if both $s$ and $s^{\prime}$ are.

Proof. (ii) Assume that $s$ and $s^{\prime}$ are primary. Consider any $x \in \operatorname{adm}(s) \cap \operatorname{adm}\left(s^{\prime}\right)$. By definition, $h, h^{\prime} \in H_{x}$ for some $h \in a d h(s)$ and $h^{\prime} \in a d h\left(s^{\prime}\right)$. Suppose for reductio that $x \notin \operatorname{adm}\left(s^{*}\right)$. Then $\operatorname{adh}\left(s^{*}\right) \cap H_{x}=\varnothing$, and hence by (i) and Proposition 6.2, $y \notin \operatorname{dom}\left(s^{*}\right)=\operatorname{dom}(s) \cap \operatorname{dom}\left(s^{\prime}\right)$ for each $y \geqslant x$, and consequently, since $h \notin \operatorname{adh}\left(s^{*}\right)$, there is a $z \in h \cap \operatorname{dom}\left(s^{*}\right)$ such that $h \notin s^{*}(z)$ and $z<x$. By definition, $s^{*}(z)=s(z) \cap s^{\prime}(z)$. Then $h \in a d h(s) \cap H_{x}$ implies $h \in s(z)-s^{\prime}(z)$, and $h^{\prime} \in \operatorname{adh}\left(s^{\prime}\right) \cap H_{x}$ implies $h^{\prime} \in s^{\prime}(z)$, and then by NC, $H_{x} \subseteq s^{\prime}(z)$, and hence $h \in s^{\prime}(z)$, a contradiction.

(iii) Let $s$ and $s^{\prime}$ be backward closed, and $s^{\prime}$ complete, in $M$. Suppose for reductio that $h \in \operatorname{adh}\left(s^{*}\right)-\operatorname{adh}(s)$, i.e., $h \notin s(x)$ for an $x \in \operatorname{dom}(s) \cap h$, and

$$
h \in s^{*}(y) \subseteq s^{\prime}(y) \text { for each } y \in c,
$$


where $c=\operatorname{dom}\left(s^{*}\right) \cap h$. We first claim that

$$
x \notin \operatorname{dom}\left(s^{\prime}\right),
$$

for otherwise we have $x \in c$ since $x \in \operatorname{dom}(s) \cap h$, and then $h \in s^{*}(x) \subseteq s(x)$, a contradiction. Next, suppose for reductio that $x^{\prime} \notin \operatorname{dom}(s)$ for an $x^{\prime} \in \operatorname{dom}\left(s^{\prime}\right) \cap h$. Since $x, x^{\prime} \in h$, either $x^{\prime}<x$ or $x \leqslant x^{\prime}$, and then, since $s$ is backward closed in $M, x^{\prime}<x$ only if $x^{\prime} \in \operatorname{dom}(s)$, contrary to the supposition of this reductio, and hence $x \leqslant x^{\prime}$. But $s^{\prime}$ is also backward closed in $M$, and hence $x \in \operatorname{dom}\left(s^{\prime}\right)$, contrary to (8). We conclude from this reductio that $\operatorname{dom}\left(s^{\prime}\right) \cap h \subseteq \operatorname{dom}(s)$, and then $c=\operatorname{dom}(s) \cap \operatorname{dom}\left(s^{\prime}\right) \cap h=\operatorname{dom}\left(s^{\prime}\right) \cap h$, and hence by (7), $h \in \operatorname{adh}\left(s^{\prime}\right)$. But $s^{\prime}$ is complete in $M$, and hence $h \cap M \subseteq \operatorname{dom}\left(s^{\prime}\right)$, contrary to (8). The rest of (iii) is straightforward.

(iv) follows from (i) and (iii). (v) For each $H \in O u t c m B d r_{M}$, that $H \in \operatorname{ado}_{M}(s \sqcap$ $\left.s^{\prime}\right)$ is equivalent to each of the following:

- $H \subseteq \bigcup \operatorname{Uado}_{M}\left(s \sqcap s^{\prime}\right)$

Fact 4.2

- $H \subseteq \operatorname{adh}\left(s \sqcap s^{\prime}\right) \cap H_{\langle M\rangle}$

Proposition 4.6

- $H \subseteq \operatorname{adh}(s) \cap \operatorname{adh}\left(s^{\prime}\right) \cap H_{\langle M\rangle}$

- $H \subseteq\left(\bigcup \operatorname{ado}_{M}(s)\right) \cap\left(\operatorname{Uado}_{M}\left(s^{\prime}\right)\right)$

- $H \in \operatorname{ado}_{M}(s) \cap \operatorname{ado}_{M}(s)$

Proposition 4.6

Fact 4.2

(i) and (vi)-(x) are easily verifiable by definition and (ii)-(iii).

Note that Proposition 6.3 (iii-vi) have little room for a generalization concerning the completeness requirement for strategies. In other words, $\operatorname{adh}\left(s \sqcap s^{\prime}\right) \subseteq \operatorname{adh}(s)$ may fail if $s^{\prime}$ is not complete in $M .^{17}$

Let $\mathcal{E}$ be any group. For each $s \in C P$-Strategy $y_{\mathcal{E}}^{M}$ and each group $\mathcal{G} \subseteq \mathcal{E}$, let $\left.s\right|_{\mathcal{G}}$ be the strategy for $\mathcal{G}$ in $M$ such that $\operatorname{dom}\left(\left.s\right|_{\mathcal{G}}\right)=\operatorname{dom}(s)$ and for each $x \in \operatorname{dom}(s)$, $\left.s(x) \subseteq s\right|_{\mathcal{G}}(x)$, i.e., $\left.s\right|_{\mathcal{G}}(x)$ is the only member of Choice $_{\mathcal{G}}^{x}$ that includes $s(x)$. We call $\left.s\right|_{\mathcal{G}}$ the subordinate strategy for $\mathcal{G}$ in $s$. Note that because $s \in C P$-Strategy $\mathcal{E}_{\mathcal{E}}^{M}$, $\left.s\right|_{\mathcal{G}}$ is obviously backward closed in $M$, and completely admits every $h \in \operatorname{adh}(s)$, and hence is primary. Note also that because $a d h(s)$ and $a d h\left(\left.s\right|_{\mathcal{G}}\right)$ may not in general be the same, $\left.s\right|_{\mathcal{G}}$ may not in general be a complete strategy for $\mathcal{G}$.

Proposition 6.4. (AC). Let $\mathcal{F}$ and $\mathcal{G}$ be disjoint groups and $\mathcal{E}=\mathcal{F} \cup \mathcal{G}$, let $s \in C P$ Strategy $y_{\mathcal{E}}^{M}$, and let $s_{\mathcal{F}}$ and $s_{\mathcal{G}}$ be any complete primary extensions of $s \mid \mathcal{F}$ and $\left.s\right|_{\mathcal{G}}$ in $M$ respectively. Then $\operatorname{adh}\left(s_{\mathcal{F}}\right) \cap a d h\left(s_{\mathcal{G}}\right)=\operatorname{adh}(s)$ and $s=\left.\left.s\right|_{\mathcal{F}} \sqcap s\right|_{\mathcal{G}}=s_{\mathcal{F}} \sqcap s_{\mathcal{G}}=$ $\left.s\right|_{\mathcal{F}} \sqcap s_{\mathcal{G}}=\left.s_{\mathcal{F}} \sqcap s\right|_{\mathcal{G}}$.

Proof. It follows by definition that $s=\left.\left.s\right|_{\mathcal{F}} \sqcap s\right|_{\mathcal{G}}$. Suppose that $h \in \operatorname{adh}(s)$. Because $s$ completely admits $h$ in $M, h \in s(x)$ for each $x \in h \cap M$, and then, because

\footnotetext{
${ }^{17}$ Let $x<y$, let Choice $_{\beta}^{y}=\left\{K, K^{\prime}\right\}$ with $K \neq K^{\prime}$, and let $h \in K$ and $h^{\prime} \in K^{\prime}$. Suppose that $h, h^{\prime} \in K_{\alpha} \cap K_{\beta}$ for a $K_{\alpha} \in$ Choice $_{\alpha}^{x}$ and a $K_{\beta} \in$ Choice $_{\beta}^{x}$ (the rest of the choice situation is not essential). Let $s_{\alpha}$ and $s_{\beta}$ be strategies for $\alpha$ and $\beta$ in $\{x, y\}$ such that $\operatorname{dom}\left(s_{\alpha}\right)=\{x\}$ and $\operatorname{dom}\left(s_{\beta}\right)=\{x, y\}, s_{\alpha}(x)=K_{\alpha}$ and $s_{\beta}(x)=K_{\beta}$, and $s_{\beta}(y)=K^{\prime}$. It is then easy to verify that $h \in \operatorname{adh}\left(s_{\alpha} \sqcap s_{\beta}\right)$ but $h \notin \operatorname{adh}\left(s_{\beta}\right)$.
} 
$s(x)=\left.\left.s\right|_{\mathcal{F}}(x) \cap s\right|_{\mathcal{G}}(x)$ for each such $x, h \in s_{\mathcal{F}}(x) \cap s_{\mathcal{G}}(x)$ for each $x \in h \cap M$, and hence $h \in \operatorname{adh}\left(s_{\mathcal{F}}\right) \cap \operatorname{adh}\left(s_{\mathcal{G}}\right)$. Suppose for reductio that $h \in \operatorname{adh}\left(s_{\mathcal{F}}\right) \cap \operatorname{adh}\left(s_{\mathcal{G}}\right)$ and $h \notin \operatorname{adh}(s)$. Then $h \notin s(x)$ for an $x \in h \cap \operatorname{dom}(s)$, and $h \in s_{\mathcal{F}}(x) \cap s_{\mathcal{G}}(x)$ because $\operatorname{dom}(s) \subseteq \operatorname{dom}\left(s_{\mathcal{F}}\right) \cap \operatorname{dom}\left(s_{\mathcal{G}}\right)$. By definition, $x \in \operatorname{dom}(s)$ implies $s_{\mathcal{F}}(x)=\left.s\right|_{\mathcal{F}}(x)$ and $s_{\mathcal{G}}(x)=\left.s\right|_{\mathcal{G}}(x)$, and hence $\left.\left.h \in s\right|_{\mathcal{F}}(x) \cap s\right|_{\mathcal{G}}(x)=s(x)$, a contradiction. It follows that $\operatorname{adh}\left(s_{\mathcal{F}}\right) \cap a d h\left(s_{\mathcal{G}}\right)=\operatorname{adh}(s)$. By Proposition 6.3(iv,viii,ix), $\operatorname{adh}\left(s_{\mathcal{F}} \sqcap\right.$ $\left.s_{\mathcal{G}}\right)=\operatorname{adh}\left(s_{\mathcal{F}}\right) \cap a d h\left(s_{\mathcal{G}}\right)$, and both $s_{\mathcal{F}} \sqcap s_{\mathcal{G}}$ and $s$ are primary and complete along their admitted histories in $M$, and hence $\operatorname{dom}\left(s_{\mathcal{F}} \sqcap s_{\mathcal{G}}\right)=\operatorname{dom}(s)$, from which it follows that $s_{\mathcal{F}} \sqcap s_{\mathcal{G}}=s$. The rest of the proposition is guaranteed by definition.

\section{Distinguishability}

Let $\mathcal{G}$ be any group, and let $m$ be any point. Speaking in an abstract way, what $\mathcal{G}$ can do at $m$ is identified with a set of histories within which $\mathcal{G}$ may intuitively constrain the future course of events to lie, as suggested in Belnap et al. (2001) and Horty (2001). Such a set of histories is, of course, presented as a member of Choice $_{\mathcal{G}}^{m}$. To put the matter in a different way, we may say that what $\mathcal{G}$ can do at best at a moment is identified with a maximal set of histories indistinguishable for $\mathcal{G}$ at the moment, where $h$ and $h^{\prime}$ are distinguishable for $\mathcal{G}$ at $m$ if $h, h^{\prime} \in H_{m}$ and Choice $_{\mathcal{G}}^{m}(h) \neq$ Choice $_{\mathcal{G}}^{m}\left(h^{\prime}\right) .{ }^{18}$ Concerning what $\mathcal{G}$ can do at a single point, this notion of distinguishability may not seem to provide more than what the notion of choice does, for a maximal set of histories indistinguishable for $\mathcal{G}$ at $m$ is nothing but a member of Choice $_{\mathcal{G}}^{m}$. Concerning what $\mathcal{G}$ can do through a field, nevertheless, the notion of distinguishability does provide more than that of choice. By making choices at various points in a field $M, \mathcal{G}$ may also constrain the future course of events to lie within a set of histories passing through $M$. Applying the notion of distinguishability, we may differentiate one from another of what $\mathcal{G}$ can do through $M$, which amounts to identifying each of them with a maximal set of histories indistinguishable for $\mathcal{G}$. Metaphorically speaking, distinguishability displays what $\mathcal{G}$ can do through $M$ at the highest resolution. In this section, we study what groups can do through a field in terms of distinguishability.

Let $M$ be any field, let $\mathcal{G}$ be any group, and let $h, h^{\prime} \in H_{\langle M\rangle} . h$ and $h^{\prime}$ are distinguishable for $\mathcal{G}$ in $M$ if they are distinguishable for $\mathcal{G}$ at a point in $M$, and are indistinguishable for $\mathcal{G}$ in $M$ otherwise. Intuitively, two histories are distinguishable for $\mathcal{G}$ in $M$ just in case some choices for $\mathcal{G}$ at a point in $M$ can tell them apart. It is easy to see that the relation of distinguishability for $\mathcal{G}$ in $M$ is irreflexive and symmetrical, while the relation of indistinguishability for $\mathcal{G}$ in $M$ is reflexive and symmetrical. Note that for $h$ and $h^{\prime}$ to be distinguishable for $\mathcal{G}$ at $x$, they must both pass through $x$. In other words, distinguishability requires availability. It should then be clear that for $h$ and $h^{\prime}$ to be distinguishable for $\mathcal{G}$ at $m$, it is not enough to only have that $h^{\prime} \notin$ Choice $_{\mathcal{G}}^{x}(h)$.

18 The idea here of distinguishability is from Belnap. See, e.g., Belnap (1991). 
For each $H \subseteq H_{\langle M\rangle}, H$ is $\mathcal{G}$-indistinguishable in $M$ if members of $H$ are pairwise indistinguishable for $\mathcal{G}$ in $M$. When the field $M$ is clear in the context, we often drop the phrase "in $M$ ". The following proposition shows that histories distinguishable for a group are distinguishable for all its super-groups, or by contraposition, histories indistinguishable for a group are indistinguishable for all its sub-groups.

Proposition 7.1. Let $M$ be any field, let $\mathcal{F}$ and $\mathcal{G}$ be any groups such that $\mathcal{F} \subseteq \mathcal{G}$, and let $h, h^{\prime} \in H_{\langle M\rangle}$ and $H \subseteq H_{\langle M\rangle}$. Then

(i) if $h$ and $h^{\prime}$ are distinguishable for $\mathcal{F}$, so are they for $\mathcal{G}$; and

(ii) if $H$ is $\mathcal{G}$-indistinguishable, it is $\mathcal{F}$-indistinguishable.

Proof. (i) Suppose that $h$ and $h^{\prime}$ are distinguishable for $\mathcal{F}$. Then there is an $m \in M$ such that $h, h^{\prime} \in H_{m}$ and Choice $_{\mathcal{F}}^{m}(h) \neq$ Choice $_{\mathcal{F}}^{m}\left(h^{\prime}\right)$. Since $\mathcal{F} \subseteq \mathcal{G}$, Choice $_{\mathcal{G}}^{m}(h) \subseteq$ Choice $_{\mathcal{F}}^{m}(h)$ and Choice $_{\mathcal{G}}^{m}\left(h^{\prime}\right) \subseteq$ Choice $_{\mathcal{F}}^{m}\left(h^{\prime}\right)$, and hence, since Choice $_{\mathcal{F}}^{m}(h) \cap$ Choice $_{\mathcal{F}}^{m}\left(h^{\prime}\right)=\varnothing$, Choice $_{\mathcal{G}}^{m}(h) \neq$ Choice $_{\mathcal{G}}^{m}\left(h^{\prime}\right)$. It then follows that $h$ and $h^{\prime}$ are distinguishable for $\mathcal{G}$. (ii) follows directly from (i).

Provided that $A$ is a nonempty set, a classification of $A$ is a set-theoretical "cover" of $A$, i.e., a subset $\mathbb{X}$ of $\mathscr{P}(A)$ (the powerset of $A$ ) such that $\bigcup \mathbb{X}=A$ and for all $X, X^{\prime} \in \mathbb{X}, X \subseteq X^{\prime}$ only if $X=X^{\prime}$. A classification of $A$ is like a partition of $A$, as they both satisfy the condition of exhaustiveness, i.e., $\cup \mathbb{X}=A$. The difference between them is obvious, too. A classification allows its members to partly overlap, whereas a partition needs to satisfy the condition of disjointedness, i.e., its members need to be pairwise disjoint. A classification $\mathbb{X}$ of $A$ is trivial if $\mathbb{X}=\{A\}$. Note that if $\mathbb{X}$ is a non-trivial classification of $A$, then $\bigcap \mathbb{X}$ cannot be a member of $\mathbb{X}$ because no member of a classification is a proper subset of another. For the same reason, $\varnothing$ is never a member of any classification of any nonempty set.

Let $M$ be any field, and let $H \subseteq H_{\langle M\rangle}$. For each group $\mathcal{G}, H$ is a maximal $\mathcal{G}$-indistinguishable set (of histories) through $M$ (a $\mathcal{G}$-MIS through $M$ ) if $H$ is $\mathcal{G}$-indistinguishable in $M$ but no proper extension of $H$ in $H_{\langle M\rangle}$ is. We will drop the phrase "through $M$ " when $M$ is clear in the context. Note that a $\mathcal{G}$-MIS through $M$ is a maximal set of histories that $\mathcal{G}$ cannot distinguish in $M$, and is therefore a minimal set of histories within which $\mathcal{G}$ can constrain the future course of events to lie, i.e., one of what $\mathcal{G}$ can do at best through $M$.

For each group $\mathcal{G}$, let $\mathbb{A}_{M, \mathcal{G}}$ be the set of all $\mathcal{G}$-MISs through $M$. Applying an argument similar to that used in Lindenbaum's lemma, one can easily verify that for each $h \in H_{\langle M\rangle}$ and each group $\mathcal{G}, h$ is contained in a $\mathcal{G}$-MIS through $M$. Hence we have the following:

Fact 7.2. (AC). For each field $M$ and each group $\mathcal{G}, \mathbb{A}_{M, \mathcal{G}}$ is a classification of $H_{\langle M\rangle}$.

For each group $\mathcal{G}$, let us call the classification $\mathbb{A}_{M, \mathcal{G}}$ of $H_{\langle M\rangle}$ the classification of $H_{\langle M\rangle}$ determined by $\mathcal{G}$. The following proposition provides a correspondence between $\mathcal{G}$-MISs and complete primary strategies for $\mathcal{G}$ : a $\mathcal{G}$-MIS through $M$ is nothing but the set of histories in $H_{\langle M\rangle}$ admitted by a complete primary strategy $s$ for $\mathcal{G}$ in $M$. Consequently, members of the classification of $H_{\langle M\rangle}$ determined by $\mathcal{G}$ are sets of histories in $H_{\langle M\rangle}$ admitted by complete primary strategies for $\mathcal{G}$ in $M$. 
Proposition 7.3. Let $\mathcal{G}$ be any group, let $M$ be any field, and let $H \subseteq H_{\langle M\rangle}$. Then $H$ is a $\mathcal{G}$-MIS through $M$ iff $H=\operatorname{adh}(s) \cap H_{\langle M\rangle}$ for an $s \in C P$-Strategy $\mathcal{G}_{\mathcal{G}}^{M}$. Hence $\mathbb{A}_{M, \mathcal{G}}=\left\{\operatorname{adh}(s) \cap H_{\langle M\rangle}: s \in C P\right.$-Strategy $\left.y_{\mathcal{G}}^{M}\right\}$.

Proof. Suppose that $H$ is a $\mathcal{G}$-MIS. Let $D=M \cap(\bigcup H)$. We claim that

$$
\text { for each } x \in D, K \cap H \neq \varnothing \text { for exactly one } K \in \text { Choice }_{\mathcal{G}}^{x} \text {. }
$$

Let $x \in D$. Then $x \in h \cap M$ for an $h \in H$. Letting $K=$ Choice $_{\mathcal{G}}^{x}(h)$, we have that $K \cap H \neq \varnothing$. For each $K^{\prime} \in$ Choice $_{\mathcal{G}}^{x}$ such that $K^{\prime} \cap H \neq \varnothing, K^{\prime}=$ Choice $_{\mathcal{G}}^{x}\left(h^{\prime}\right)$ for an $h^{\prime} \in H$, and hence, since $H$ is $\mathcal{G}$-indistinguishable, Choice $\mathcal{G}_{\mathcal{G}}^{x}\left(h^{\prime}\right)=K$. It then follows that (9) holds. Now let $s$ be a function on $D$ such that for each $x \in D$, $s(x)=$ the only $K \in$ Choice $_{\mathcal{G}}^{x}$ such that $K \cap H \neq \varnothing$. Then $s$ is a strategy for $\mathcal{G}$ in $M$ that is backward closed in $M$. We show below that $H=a d h(s) \cap H_{\langle M\rangle}$ and $s \in C P$-Strategy ${ }_{\mathcal{G}}^{M}$.

Consider any $h \in H_{\langle M\rangle}$. If $h \in H$, then for each $x \in h \cap \operatorname{dom}(s), s(x)$ is the only $K \in$ Choice $_{\mathcal{G}}^{x}$ such that $K \cap H \neq \varnothing$, and hence $h \in s(x)$, from which it follows that $h \in \operatorname{adh}(s)$. If $h \in H_{\langle M\rangle}-H$, then, since $H$ is a $\mathcal{G}$-MIS, there is an $h^{\prime} \in H$ and a $y \in M$ such that $h, h^{\prime} \in H_{y}$ and Choice $_{\mathcal{G}}^{y}(h) \neq$ Choice $_{\mathcal{G}}^{y}\left(h^{\prime}\right)$, and hence by definition of $s, s(y)=$ Choice $_{\mathcal{G}}^{y}\left(h^{\prime}\right)$, and consequently $h \notin a d h(s)$. It then follows that $H=\operatorname{adh}(s) \cap H_{\langle M\rangle}$, which implies that $\operatorname{dom}(s)=M \cap(\bigcup H) \subseteq \bigcup a d h(s)$ and that $M \cap h \subseteq \operatorname{dom}(s)$ for each $h \in a d h(s)$, and hence $s$ is primary and is complete in $M$.

Next suppose that $s \in C P$-Strategy $y_{\mathcal{G}}^{M}$. We show that $H$ is an $\mathcal{G}$-MIS with $H=$ $\operatorname{adh}(s) \cap H_{\langle M\rangle}$. To show that $H$ is $\mathcal{G}$-indistinguishable, it suffices to let $h \in H$ and $h^{\prime} \in H_{\langle M\rangle}$ such that $K=$ Choice $_{\mathcal{G}}^{x}(h) \neq$ Choice $_{\mathcal{G}}^{x}\left(h^{\prime}\right)=K^{\prime}$ for an $x \in M$, and show that $h^{\prime} \notin a d h(s)$. Because $h \in \operatorname{adh}(s) \cap K, s(x)=K$ by the completeness of $s$, and then, since $h^{\prime} \in K^{\prime} \neq K, h^{\prime} \notin a d h(s)$. To show further that $H$ is a $\mathcal{G}$-MIS, consider any $h_{0} \in H_{\langle M\rangle}-a d h(s)$. By definition, $h_{0} \notin s(y)$ for a $y \in h_{0} \cap \operatorname{dom}(s)$, and hence Choice $e_{\mathcal{G}}^{y}\left(h_{0}\right) \neq s(y)$. Because $s$ is primary and complete in $M$, there is an $h^{\prime} \in \operatorname{adh}(s)$ such that $s(y)=$ Choice $_{\mathcal{G}}^{y}\left(h^{\prime}\right)$. Since $h^{\prime} \in H_{y} \subseteq H_{\langle M\rangle}$, it follows that $H \cup\left\{h_{0}\right\}$ is not $\mathcal{G}$-indistinguishable. Hence $H$ is an $\mathcal{G}$-MIS.

As noted earlier, what a group can do at best through a field $M$ is to attain a maximal set of histories indistinguishable for the group in $M$. Proposition 7.3 provides a "finest" characterization of what a group can do through $M$ : For each group $\mathcal{G}$, to attain a $\mathcal{G}$-MIS $H$ means the same as coordinating its members' efforts in such a way that their joint choices form a complete primary strategy that admits $H$.

The following proposition shows a relation between the classification of $H_{\langle M\rangle}$ determined by a group and the classifications determined by its sub-groups, which will be useful in our discussion of independence.

Proposition 7.4. (AC). Let $M$ be any field, and let $\mathcal{F}$ and $\mathcal{G}$ be disjoint groups. Then $\mathbb{A}_{M, \mathcal{F} \cup \mathcal{G}}=\left\{H \cap H^{\prime}: H \in \mathbb{A}_{M, \mathcal{F}} \wedge H^{\prime} \in \mathbb{A}_{M, \mathcal{G}}\right\}$. 
Proof. Consider any $H \in \mathbb{A}_{M, \mathcal{F}}$ and $H^{\prime} \in \mathbb{A}_{M, \mathcal{G}}$. By Proposition 7.3, $H=\operatorname{adh}(s) \cap$ $H_{\langle M\rangle}$ and $H^{\prime}=\operatorname{adh}\left(s^{\prime}\right) \cap H_{\langle M\rangle}$ for some $s \in C P$-Strategy ${ }_{\mathcal{F}}^{M}$ and $s^{\prime} \in C P$-Strategy $y_{\mathcal{G}}^{M}$, and then by Fact 5.4 (iii), $\operatorname{dom}(s) \cap \operatorname{dom}\left(s^{\prime}\right) \neq \varnothing$, and hence $H \cap H^{\prime}=\operatorname{adh}\left(s \sqcap s^{\prime}\right) \cap$ $H_{\langle M\rangle}$ by Proposition 6.3(iv). We know by Proposition 6.3 (viii,x) that $s \sqcap s^{\prime} \in C P$ -

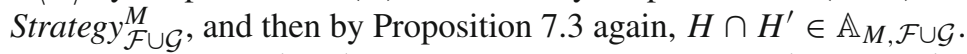

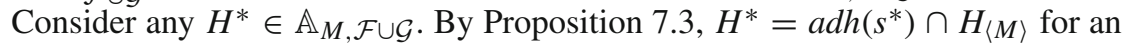
$s^{*} \in C P$-Strategy $y_{\mathcal{F} \cup \mathcal{G}}^{M}$. Let $s_{\mathcal{F}}$ and $s_{\mathcal{G}}$ be any complete primary extensions of $s^{*} \mid \mathcal{F}$ and $\left.s^{*}\right|_{\mathcal{G}}$ in $M$ respectively. By Proposition 6.4, $s^{*}=s_{\mathcal{F}} \sqcap s_{\mathcal{G}}$, and then by Proposition 6.3(iv), $\operatorname{adh}\left(s_{\mathcal{F}}\right) \cap \operatorname{adh}\left(s_{\mathcal{G}}\right) \cap H_{\langle M\rangle}=H^{*}$, while $\operatorname{adh}\left(s_{\mathcal{F}}\right) \cap H_{\langle M\rangle} \in \mathbb{A}_{M, \mathcal{F}}$ and $\operatorname{adh}\left(s_{\mathcal{G}}\right) \cap H_{\langle M\rangle} \in \mathbb{A}_{M, \mathcal{G}}$ by Proposition 7.3.

When considering what groups can do in a field, we sometime want to identify such doings with sets of outcomes bordering the field rather than sets of histories passing through the field. In such cases, distinguishability may also be applied to outcomes bordering the field. Let $\mathcal{G}$ be any group. For each point $x$, outcomes $H$ and $H^{\prime}$ are distinguishable for $\mathcal{G}$ at $x$ if there are distinct $K, K^{\prime} \in$ Choice $_{\mathcal{G}}^{x}$ such that $H \subseteq K$ and $H^{\prime} \subseteq K^{\prime}$. Let $M$ be any properly covered field, and let $H, H^{\prime} \in O u t c m B d r_{M}$. $H$ and $H^{\prime}$ are distinguishable for $\mathcal{G}$ in $M$ if they are distinguishable for $\mathcal{G}$ at a point in $M$, and are indistinguishable for $\mathcal{G}$ in $M$ otherwise. Note that for $H$ and $H^{\prime}$ to be distinguishable for $\mathcal{G}$ at $x$, they must be both available as possible future outcomes relative to $x$. Let $U \subseteq O \operatorname{OutcmBdr}_{M}$. $U$ is $\mathcal{G}$-indistinguishable in $M$ if all members of $U$ are pairwise indistinguishable for $\mathcal{G}$ in $M$. $U$ is a maximal $\mathcal{G}$-indistinguishable set (of outcomes) bordering $M$ (a $\mathcal{G}$-MIS bordering $M$ ) if $U$ is $\mathcal{G}$-indistinguishable in $M$ but no proper extension of $U$ in $O u t c m B d r_{M}$ is. We may drop the phrases "in $M$ " and "bordering $M$ " when $M$ is clear in the context. For each group $\mathcal{G}$, let $\mathbb{C}_{M, \mathcal{G}}$ be the set of all $\mathcal{G}$-MISs bordering $M$.

The follow proposition proves useful in our upcoming discussions.

Proposition 7.5. Let $M$ be a properly covered field, let $\mathcal{G}$ be a group, and let $U, U^{\prime} \subseteq$ OutcmBdr $_{M}$ and $H, H^{\prime} \in$ OutcmBdr $_{M}$ with $h \in H$ and $h^{\prime} \in H^{\prime}$. Then the following hold:

(i) $H$ and $H^{\prime}$ are distinguishable for $\mathcal{G}$ in $M$ iff $h$ and $h^{\prime}$ are distinguishable for $\mathcal{G}$ in $M$;

(ii) $U \in \mathbb{C}_{M, \mathcal{G}}$ iff $\bigcup U \in \mathbb{A}_{M, \mathcal{G}}$;

(iii) if $U \in \mathbb{C}_{M, \mathcal{G}}$, then $\bigcup U^{\prime} \subseteq \bigcup U$ iff $U^{\prime} \subseteq U$.

Proof. (i) holds by definition and Fact 3.2. (ii) Suppose first that $U \in \mathbb{C}_{M, \mathcal{G}}$. Then $\bigcup U$ is $\mathcal{G}$-indistinguishable by (i). To show that no proper extension of $\bigcup U$ in $H_{\langle M\rangle}$ is $\mathcal{G}$-indistinguishable, consider any $h \in H_{\langle M\rangle}-\bigcup U$. By Propositions 3.3-3.4, we let $H=\operatorname{OutcmBdr}_{M}(h)$. Since $h \in H$ and $h \notin \bigcup U, H \notin U$, and then, since $U \in \mathbb{C}_{M, \mathcal{G}}$, there is a $H^{\prime} \in U$ such that $H$ and $H^{\prime}$ are distinguishable for $\mathcal{G}$, and hence, letting $h^{\prime} \in H^{\prime}, h$ and $h^{\prime}$ are by (i) distinguishable for $\mathcal{G}$. Hence $\bigcup U \in \mathbb{A}_{M, \mathcal{G}}$. Next suppose that $\bigcup U \in \mathbb{A}_{M, \mathcal{G}}$. Then $U$ is $\mathcal{G}$-indistinguishable by (i). To show that no proper extension of $U$ in $\operatorname{OutcmBdr}_{M}$ is $\mathcal{G}$-indistinguishable, consider any $H \in O$ OutcmBdr $r_{M}$. If $H \notin U$, Proposition 3.3 implies that $H \cap(\bigcup U)=\varnothing$, and then, 
letting $h \in H$ and $h^{\prime} \in \bigcup U, h$ and $h^{\prime}$ are distinguishable for $\mathcal{G}$ by our supposition, and hence by (i), $H$ and some member of $U$ are distinguishable for $\mathcal{G}$.

(iii) Suppose that $\bigcup U^{\prime} \subseteq \bigcup U$ with $U \in \mathbb{C}_{M, \mathcal{G}}$. Then $\bigcup U \in \mathbb{A}_{M, \mathcal{G}}$ by (ii). Now suppose for reductio that $H \in U^{\prime}-U$. Then there is an $H^{\prime} \in U$ such that $H$ and $H^{\prime}$ are distinguishable for $\mathcal{G}$ in $M$. Letting $h \in H \subseteq \bigcup U^{\prime}$ and $h^{\prime} \in H^{\prime} \subseteq \bigcup U$, we know by (i) that $h$ and $h^{\prime}$ are distinguishable for $\mathcal{G}$ in $M$, and hence $h \notin \bigcup U$, contrary to that $\bigcup U^{\prime} \subseteq \bigcup U$. Hence $U^{\prime} \subseteq U$.

Similar to Proposition 7.1 and Fact 7.2, we have the following:

Proposition 7.6. Let $M$ be any field, let $\mathcal{F}$ and $\mathcal{G}$ be any groups such that $\mathcal{F} \subseteq \mathcal{G}$, and let $H, H^{\prime} \in O u t c m B d r_{M}$ and $U \subseteq O u t c m B d r_{M}$. Then

(i) if $H$ and $H^{\prime}$ are distinguishable for $\mathcal{F}$, so are they for $\mathcal{G}$; and

(ii) if $U$ is $\mathcal{G}$-indistinguishable, it is $\mathcal{F}$-indistinguishable.

Proof. Apply Propositions 7.1 and 7.5(i).

Fact 7.7. (AC). For each properly covered field $M$ and each group $\mathcal{G}, \mathbb{C}_{M, \mathcal{G}}$ is a classification of $\mathrm{OutcmBd} r_{M}$.

The following proposition shows that a $\mathcal{G}$-MIS bordering $M$ is nothing but the set $\operatorname{ado}_{M}(s)$ for a complete primary strategy $s$ for $\mathcal{G}$ in $M$.

Proposition 7.8. Let $\mathcal{G}$ be any group, let $M$ be any properly covered field, and let $U \subseteq O u t c m B d r_{M}$. Then $U$ is a $\mathcal{G}$-MIS bordering $M$ iff $U=\operatorname{ado}_{M}(s)$ for an $s \in C P$-Strategy $y_{\mathcal{G}}^{M}$. Hence $\mathbb{C}_{M, \mathcal{G}}=\left\{\operatorname{ado}_{M}(s): s \in C P\right.$-Strategy $\left.{ }_{\mathcal{G}}^{M}\right\}$.

Proof. Let $U \subseteq$ OutcmBdr $r_{M}$ and $H=\bigcup U$. Then that $U \in \mathbb{C}_{M, \mathcal{G}}$ is equivalent to each of the following:

- $H \in \mathbb{A}_{M, \mathcal{G}}$,

- $H=\operatorname{adh}(s) \cap H_{\langle M\rangle}$ for an $s \in C P$-Strategy ${ }_{\mathcal{G}}^{M}$,

Proposition 7.5(ii)

- $H=\bigcup \operatorname{ado}_{M}(s)$ for an $s \in C P$-Strategy $y_{\mathcal{G}}^{M}$,

Proposition 7.3

- $U=\operatorname{ado}_{M}(s)$ for an $s \in C P$-Strategy ${ }_{\mathcal{G}}^{M}$.

Proposition 4.6

Hence the conclusion holds.

The following is a simple consequence of Propositions 7.3 and 7.8.

Proposition 7.9. Let $M$ be a properly covered field, let $\mathcal{G}$ be a group, and let $H \subseteq$ $H_{\langle M\rangle}$. Then $H \in \mathbb{A}_{M, \mathcal{G}}$ iff $H=\bigcup U$ for a $U \in \mathbb{C}_{M, \mathcal{G}}$.

Proof. $H \in \mathbb{A}_{M, \mathcal{G}}$ iff (by Proposition 7.3) $H=a d h(s) \cap H_{\langle M\rangle}$ for an $s \in C P$ Strategy $y_{\mathcal{G}}^{M}$ iff (by Proposition 4.6) $H=\bigcup \operatorname{Uado}_{M}(s)$ for an $s \in C P$-Strategy $y_{\mathcal{G}}^{M}$ iff (by Proposition 7.8) $H=\bigcup U$ for a $U \in \mathbb{C}_{M, \mathcal{G}}$.

The idea in the following proposition is similar to that in Proposition 7.4, but with respect to future outcomes rather than histories. 
Proposition 7.10. (AC). Let $M$ be any properly covered field, and let $\mathcal{F}$ and $\mathcal{G}$ be disjoint groups. Then $\mathbb{C}_{M, \mathcal{F} \cup \mathcal{G}}=\left\{U^{\prime} \cap U^{\prime \prime}: U^{\prime} \in \mathbb{C}_{M, \mathcal{F}} \wedge U^{\prime \prime} \in \mathbb{C}_{M, \mathcal{G}}\right\}$.

Proof. Let $U \subseteq$ OutcmBdr $r_{M}$ and $H=\bigcup U$. Then $U \in \mathbb{C}_{M, \mathcal{F} \cup \mathcal{G}}$ iff (by Proposition 7.5(ii)) $H \in \mathbb{A}_{M, \mathcal{F} \cup \mathcal{G}}$ iff (by Proposition 7.4) $H=H^{\prime} \cap H^{\prime \prime}$ for an $H^{\prime} \in \mathbb{A}_{M, \mathcal{F}}$ and an $H^{\prime \prime} \in \mathbb{A}_{M, \mathcal{G}}$ iff (by Propositions 7.9 and 7.5(ii))

$$
H=\left(\bigcup U^{\prime}\right) \cap\left(\bigcup U^{\prime \prime}\right) \text { for a } U^{\prime} \in \mathbb{C}_{M, \mathcal{F}} \text { and a } U^{\prime \prime} \in \mathbb{C}_{M, \mathcal{G}}
$$

It is easy to verify by Proposition 3.3 that $\left(\bigcup U^{\prime}\right) \cap\left(\bigcup U^{\prime \prime}\right)=\bigcup\left(U^{\prime} \cap U^{\prime \prime}\right)$, and then Proposition 3.3 again, (10) holds iff $U=U^{\prime} \cap U^{\prime \prime}$ for a $U^{\prime} \in \mathbb{C}_{M, \mathcal{F}}$ and a $U^{\prime \prime} \in \mathbb{C}_{M, \mathcal{G}}$.

\section{Inactivity and Busyness}

Before we move on to the notion of independence, we present a short discussion of inactivity and "busyness" in a field. This is because, as it turns out, the inactivity of a group plays a special role, often behind the curtain, in our discussion of independence, while the absence of "backward busyness" allows us to have a characterization of independence in terms of a set-theoretical relation between groups.

Let $\mathcal{G}$ be any group, $x$ any point, $X$ any set of points and $h$ any history. $\mathcal{G}$ is active at $x$, or $x$ is a (real) choice point for $\mathcal{G}(\alpha)$, if Choice $_{\mathcal{G}}^{x} \neq\left\{H_{x}\right\}$. $\mathcal{G}$ is inactive at $x$ if Choice $e_{\mathcal{G}}^{x}=\left\{H_{x}\right\}$, is inactive in $X$ if it is inactive at each $x \in X$, and is inactive along $h$ in $X$ if it is inactive in $h \cap X$. We say that an agent $\alpha$ is active/inactive at $x$, in $X$, or along $h$ in $X$, if $\{\alpha\}$ is so. It is easy to see that the empty group is always inactive in every field, and that if $\mathcal{G}$ is inactive at $x$, in $X$, or along $h$ in $X$, then so is every sub-group of $\mathcal{G}$. Furthermore we have the following list of simple facts concerning inactivity.

Fact 8.1. Let $M$ be any field, let $\mathcal{G}$ be any group, and let $s \in C P$-Strategy ${ }_{\mathcal{G}}^{M}$. Then the following hold:

(i) if $\mathcal{G}$ is inactive in $M$, then $C P$-Strategy $\mathcal{G}_{\mathcal{G}}^{M}=\{s\}, \operatorname{dom}(s)=M$ and $\operatorname{adh}(s)$ is the set of all histories;

(ii) if $M$ is properly covered and $\mathcal{G}$ is inactive in $M$, then $\operatorname{ado}_{M}(s)=\operatorname{OutcmBdr}_{M}$;

(iii) if $\mathcal{G}$ is inactive in $M$ and $s^{\prime}$ is a strategy in $M, \operatorname{adh}\left(s^{\prime}\right) \subseteq \operatorname{adh}(s)$;

(iv) if $\mathcal{G}$ is inactive in $\operatorname{dom}(s)$, then $\operatorname{dom}(s)=M$ (and hence $\mathcal{G}$ is inactive in $M$ ). (AC)

Proof. We show only (iv). Suppose that $\mathcal{G}$ is inactive in $\operatorname{dom}(s)$. Consider any $y \in M$. By the Axiom of Choice, $y \in h$ for a history $h$. Since $s(x)=H_{x}$ for each $x \in \operatorname{dom}(s)$, $h \in s(x)$ for each $x \in h \cap \operatorname{dom}(s)$, i.e., $h \in \operatorname{adh}(s)$. Because $s$ is complete in $M$, $h \cap M \subseteq \operatorname{dom}(s)$, and hence $y \in \operatorname{dom}(s)$. 
By definition, $\bigcap \mathbb{A}_{M, \mathcal{G}}$ is the set of histories in $H_{\langle M\rangle}$ each of which is indistinguishable for $\mathcal{G}$ from all histories in $H_{\langle M\rangle}$. The inactivity of a group $\mathcal{G}$ along a history $h$ in $M$, as it turns out, amounts to the indistinguishability for $\mathcal{G}$ in $M$ between $h$ and all other histories in $H_{\langle M\rangle}$. In other words, $\bigcap \mathbb{A}_{M, \mathcal{G}}=\left\{h \in H_{\langle M\rangle}: \mathcal{G}\right.$ is inactive in $h \cap M\}$, as shown below.

Proposition 8.2. Let $\mathcal{G}$ be any group, let $M$ be any field, and let $h \in H_{\langle M\rangle}$. Then $\mathcal{G}$ is inactive in $h \cap M$ iff $h \in \bigcap \mathbb{A}_{M, \mathcal{G}}$, and consequently $\mathcal{G}$ is inactive in $\left(\bigcup \cap \mathbb{A}_{M, \mathcal{G}}\right) \cap M$.

Proof. Suppose that $\mathcal{G}$ is inactive in $h \cap M$. Consider any $h^{\prime} \in H_{\langle M\rangle}$ and any $x \in M$ such that $h, h^{\prime} \in H_{x}$. Since $x \in h \cap M$, Choice $_{\mathcal{G}}^{x}=\left\{H_{x}\right\}$, and hence Choice $_{\mathcal{G}}^{x}(h)=$ Choice $_{\mathcal{G}}^{x}\left(h^{\prime}\right)$. It then follows that $h$ and $h^{\prime}$ are indistinguishable for $\mathcal{G}$ in $M$ for each $h^{\prime} \in H_{\langle M\rangle}$, and hence $h \in \bigcap \mathbb{A}_{M, \mathcal{G}}$.

Suppose next that $h \in \bigcap \mathbb{A}_{M, \mathcal{G}}$. If Choice $_{\mathcal{G}}^{x} \neq\left\{H_{x}\right\}$ for an $x \in h \cap M$, Choice $_{\mathcal{G}}^{x}(h) \neq K$ for some $K \in$ Choice $_{\mathcal{G}}^{x}$, and, since $h^{\prime} \in K$ for some $h^{\prime} \in H_{\langle M\rangle}, h$ and $h^{\prime}$ are distinguishable for $\mathcal{G}$ in $M$, contrary to the supposition that $h \in \bigcap \mathbb{A}_{M, \mathcal{G}}$. Hence $\mathcal{G}$ is inactive in $h \cap M$.

We know by Proposition 7.3 that $\bigcap \mathbb{A}_{M, \mathcal{G}} \subseteq \operatorname{adh}(s) \cap H_{\langle M\rangle}$ for every $s \in C P$ Strategy $y_{\mathcal{G}}^{M}$ (since by definition, $\bigcap \mathbb{A}_{M, \mathcal{G}} \subseteq H$ for every $H \in \mathbb{A}_{M, \mathcal{G}}$ ). Now we also know by Proposition 8.2 that for each $h \in H_{\langle M\rangle}, \mathcal{G}$ is inactive along $h$ in $M$ iff $h \in \bigcap\left\{\operatorname{adh}(s) \cap H_{\langle M\rangle}: s \in C P\right.$-Strategy $\left.{ }_{\mathcal{G}}^{M}\right\}$. That is to say, all strategies in $C P$ Strategy ${ }_{\mathcal{G}}^{M}$ "overlap" with exactly those histories in $H_{\langle M\rangle}$ that $\mathcal{G}$ is inactive along in $M$. We can similarly show the following.

Proposition 8.3. Let $\mathcal{G}$ be any group, let $M$ be any properly covered field, and let $h \in H_{\langle M\rangle}$. Then $\mathcal{G}$ is inactive in $h \cap M$ iff $\operatorname{OutcmBdr}_{M}(h) \in \cap \mathbb{C}_{M, \mathcal{G}}$, and consequently $\mathcal{G}$ is inactive in $\left(\bigcup \bigcup \cap \mathbb{C}_{M, \mathcal{G}}\right) \cap M$.

Note that $\bigcap \mathbb{A}_{M, \mathcal{G}}$ cannot be a member of $\mathbb{A}_{M, \mathcal{G}}$ if $\mathbb{A}_{M, \mathcal{G}}$ is a non-trivial classification of $H_{\langle M\rangle}$, as we noted earlier in Sect. 7. Similarly, $\bigcap \mathbb{C}_{M, \mathcal{G}}$ cannot be a member of $\mathbb{C}_{M, \mathcal{G}}$ if $\mathbb{C}_{M, \mathcal{G}}$ is a non-trivial classification of OutcmBdr $r_{M}$.

For each field $M$, a group $\mathcal{G}$ (or an agent $\alpha$ ) is sooner or later active in $M$ (SOL active in $M$ ) if for each $h \in H_{\langle M\rangle}$, there is a choice point $x \in h \cap M$ for $\mathcal{G}(\alpha)$, i.e., $\mathcal{G}$ is not inactive in $h \cap M$. Note that a group can be SOL active in $M$ when some or even all proper sub-groups of it are not. The following is a consequence of Propositions 8.2-8.3.

Corollary 8.4. For each field $M$ and each group $\mathcal{G}$, if $\mathcal{G}$ is SOL active in $M$, then $\bigcap \mathbb{A}_{M, \mathcal{G}}=\bigcap \mathbb{C}_{M, \mathcal{G}}=\varnothing$.

Now consider a complete primary strategy $s^{\prime}$ for $\mathcal{F}$ in a field $M$. It is quite possible that $\mathcal{F}$ is inactive in $M$, and then by Fact 8.1, $\operatorname{adh}(s) \subseteq \operatorname{adh}\left(s^{\prime}\right)$ for any strategy $s$ in $M$ for any group $\mathcal{G}$. In our discussion of independence, we need to deal with situations where $\operatorname{adh}(s) \subseteq \operatorname{adh}\left(s^{\prime}\right)$ holds somehow for an $s^{\prime} \in C P$-Strategy $y_{\mathcal{F}}^{M}$ and an $s \in C P$-Strategy $y_{\mathcal{G}}^{M}$ with $\mathcal{F} \cap \mathcal{G}=\varnothing$. What is a sufficient and necessary condition for $\operatorname{adh}(s) \subseteq \operatorname{adh}\left(s^{\prime}\right)$ to hold? It turns out thatthe inactivity of $\mathcal{F}$ in $M$ is not, but the 
inactivity of $\mathcal{F}$ in $\operatorname{dom}(s)$ is, such a sufficient and necessary condition, as we show below.

Proposition 8.5. Let $M$ be any field, let $\mathcal{G}$ and $\mathcal{F}$ be disjoint groups, and let $s \in C P$ Strategy $y_{\mathcal{G}}^{M}$ and $s^{\prime} \in C P$-Strategy $y_{\mathcal{F}}^{M}$. Then (i) $\mathcal{F}$ is inactive in $\operatorname{dom}(s)$ iff (ii) $\operatorname{adh}(s) \subseteq$ $\operatorname{adh}\left(s^{\prime}\right)$ iff (iii) $\operatorname{adh}(s) \cap H_{\langle M\rangle} \subseteq \operatorname{adh}\left(s^{\prime}\right)$.

Proof. Suppose that (i) holds. If $h \in \operatorname{adh}(s)-\operatorname{adh}\left(s^{\prime}\right), h \notin s^{\prime}(x)$ for some $x \in$ $h \cap \operatorname{dom}\left(s^{\prime}\right)$, and then $x \in \operatorname{dom}(s)$ because $x \in h \cap M$ and $s$ is complete along $h$ in $M$, and hence $h \in H_{x}=s^{\prime}(x)$ by (i), a contradiction. It then follows that (ii) holds, which clearly implies (iii).

Suppose that (iii) holds. We prove (i) below. We first show that

$$
\operatorname{dom}(s) \subseteq \operatorname{dom}\left(s^{\prime}\right)
$$

Consider any $x \in \operatorname{dom}(s)$. Because $s$ is primary, $h \in s(x)$ for an $h \in \operatorname{adh}(s)$, and then $h \in \operatorname{adh}(s) \cap H_{\langle M\rangle}$, and hence $h \in \operatorname{adh}\left(s^{\prime}\right)$ by (iii). Since $s^{\prime}$ is complete along $h, x \in \operatorname{dom}\left(s^{\prime}\right)$. Hence (11) holds. Now suppose for reductio that $\mathcal{F}$ is not inactive in $\operatorname{dom}(s)$. Then by (11), $s^{\prime}(y) \neq K$ for some $y \in \operatorname{dom}(s)$ and $K \in$ Choice $_{\mathcal{F}}^{y}$, and hence

$$
K \cap \operatorname{adh}\left(s^{\prime}\right)=\varnothing
$$

Because $\mathcal{F} \cap \mathcal{G}=\varnothing$, there is by $\mathrm{IA}$ an $h^{\prime} \in s(y) \cap K$. We show below that

$$
h^{\prime \prime} \in s(y) \cap K \text { for an } h^{\prime \prime} \in \operatorname{adh}(s)
$$

Assume that $h^{\prime} \notin \operatorname{adh}(s)$ (or there is nothing more to show). Then $h^{\prime} \notin s(z)$ for a $z \in h^{\prime} \cap \operatorname{dom}(s)$. Because $y, z \in h^{\prime}, z \leqslant y$ or $y<z$. We claim that

$$
y<z
$$

Since $h^{\prime} \in s(y)$ and $h^{\prime} \notin s(z), y \neq z$. Because $s$ is primary, there is an $h^{*} \in$ $s(y) \cap \operatorname{adh}(s)$, and then $y \in h^{\prime} \cap h^{*}$, and hence by NC and $h^{\prime} \notin s(z), z<y$ only if $h^{*} \notin s(z)$, contrary to that $h^{*} \in \operatorname{adh}(s)$. It follows that (14) holds. Since $s$ is primary, there is an $h^{\prime \prime} \in s(z) \cap a d h(s)$ and $z \in h^{\prime} \cap h^{\prime \prime}$. Because $h^{\prime} \in s(y) \cap K$, it follows from NC and (14) that $h^{\prime \prime} \in s(y) \cap K$, which completes the proof of (13). But $K \subseteq H_{y} \subseteq H_{\langle M\rangle}$, by which (13) implies that $\varnothing \neq K \cap \operatorname{adh}(s)=K \cap \operatorname{adh}(s) \cap H_{\langle M\rangle}$, and then by (iii), $K \cap a d h\left(s^{\prime}\right) \neq \varnothing$, contrary to (12). We then conclude from this reductio that (i) holds.

Note that clause (i) in the conclusion of Proposition 8.5 depends on no particular strategies for $\mathcal{F}$ in $M$. We then have the following as a direct consequence of Proposition 8.5. 
Corollary 8.6. Let $M$ be any field, let $\mathcal{G}$ and $\mathcal{F}$ be disjoint groups, and let $s \in$ $C P$-Strategy $\mathcal{G}_{\mathcal{G}}^{M}$ and $s^{*} \in C P$-Strategy $y_{\mathcal{F}}^{M}$ such that $\operatorname{adh}(s) \cap H_{\langle M\rangle} \subseteq \operatorname{adh}\left(s^{*}\right)$. Then $\operatorname{adh}(s) \subseteq \operatorname{adh}\left(s^{\prime}\right)$ for every $s^{\prime} \in C P$-Strategy $y_{\mathcal{F}}^{M}$.

Proof. By hypothesis and Proposition 8.5, $\mathcal{F}$ is inactive in $\operatorname{dom}(s)$, and then for each $s^{\prime} \in C P-$ Strategy $_{\mathcal{F}}^{M}, \operatorname{adh}(s) \subseteq \operatorname{adh}\left(s^{\prime}\right)$ by Proposition 8.5 again.

Recall that for each $s \in P$-Strategy ${ }_{\mathcal{G}}^{M}, \operatorname{adh}(s) \cap H_{\langle M\rangle}$ is by definition never empty.

Corollary 8.7. Let $M$ be any field, let $\mathcal{G}$ and $\mathcal{F}$ be disjoint groups, and let $\mathcal{F}$ be SOL active in $M$. Then for each $s \in C P$-Strategy $y_{\mathcal{G}}^{M}$ and each $H \in \mathbb{A}_{M, \mathcal{F}}, \operatorname{adh}(s) \cap H_{\langle M\rangle} \nsubseteq$ $H$.

Proof. Let $s \in C P$-Strategy ${ }_{\mathcal{G}}^{M}$ and $H \in \mathbb{A}_{M, \mathcal{F}}$. By Proposition 7.3, $H=\operatorname{adh}\left(s^{\prime}\right) \cap$ $H_{\langle M\rangle}$ for an $s^{\prime} \in C P$-Strategy $y_{\mathcal{F}}^{M}$. Suppose for reductio that $\operatorname{adh}(s) \cap H_{\langle M\rangle} \subseteq H \subseteq$ $\operatorname{adh}\left(s^{\prime}\right)$. Then by Corollary 8.6, $\operatorname{adh}(s) \subseteq \operatorname{adh}\left(s^{\prime \prime}\right)$, and then $\operatorname{adh}(s) \cap H_{\langle M\rangle} \subseteq$ $\operatorname{adh}\left(s^{\prime \prime}\right) \cap H_{\langle M\rangle}$, for each $s^{\prime \prime} \in C P$-Strategy $y_{\mathcal{F}}^{M}$, and hence $\varnothing \neq \operatorname{adh}(s) \cap H_{\langle M\rangle} \subseteq$ $\bigcap \mathbb{A}_{M, \mathcal{F}}$ by Proposition 7.3. This is impossible because $\mathcal{F}$ is SOL active in $M$, and hence $\bigcap \mathbb{A}_{M, \mathcal{F}}=\varnothing$ by Corollary 8.4 .

A group $\mathcal{G}$ (or an agent $\alpha$ ) is a busy chooser if there is an infinite chain of choice points for $\mathcal{G}(\alpha)$ that is both upper- and lower-bounded. ${ }^{19}$ The kind of busyness relevant to our current work is "backward busyness". $\mathcal{G}(\alpha)$ is backward busy in $M$ if there is a lower-bounded infinite chain $c$ in $M$ satisfying that for each $x \in c$, there is a $y \in c$ such that $y<x$ and Choice $_{\mathcal{G}}^{y} \neq\left\{H_{y}\right\}\left(\right.$ Choice $\left._{\alpha}^{y} \neq\left\{H_{y}\right\}\right)$. Note that when a group is infinite, the busyness of the group does not imply the busyness of any of its members or sub-groups, but if a group is not busy, neither is any of its members or sub-groups.

We know that different strategies in $M$ for the same group $\mathcal{G}$ may "overlap" in the sense of sharing some admitted histories in $H_{\langle M\rangle}$, and we do not know whether each such strategy is "disjoint" with at least one other such strategy. When $\mathcal{G}$ is SOL active but not backward busy in $M$, nevertheless, the existence of such a "disjoint" strategy is guaranteed for each complete primary strategy for $\mathcal{G}$ in $M$, as the following proposition shows.

Proposition 8.8. (AC). Let $M$ be any field, in which $\mathcal{G}$ is $\mathrm{SOL}$ active but not backward busy, and let $s \in C P$-Strategy ${ }_{\mathcal{G}}^{M}$. Then there is an $s^{\prime} \in C P$-Strategy ${ }_{\mathcal{G}}^{M}$ such that $\operatorname{adh}(s) \cap \operatorname{adh}\left(s^{\prime}\right) \cap H_{\langle M\rangle}=\varnothing .^{20}$

Proof. Let $D$ be the set of all minimal choice points for $\mathcal{G}$ in $\operatorname{dom}(s)$, i.e., the set of all choice points $x \in \operatorname{dom}(s)$ for $\mathcal{G}$ such that $y<x$ for no choice point $y \in \operatorname{dom}(s)$

\footnotetext{
${ }^{19}$ Busy choosers and busy choice sequences play a special role in various conceptual analyses of agency and technical developments, especially when achievement stit and strategies are involved. See, e.g., Belnap et al. (2001) and Xu (1995).

${ }^{20}$ The hypothesis that $s \in C P$-Strategy $y_{\mathcal{G}}^{M}$ and $\mathcal{G}$ is SOL active but not backward busy in $M$ can be weakened to that $\mathcal{G}$ is any group and $s \in C P$-Strategy $y_{\mathcal{G}}^{M}$ such that for each $h \in \operatorname{adh}(s) \cap H_{\langle M\rangle}$, there is a least choice point in $h \cap M$ for $\mathcal{G}$.
} 
for $\mathcal{G}$. For each $x \in D$, select a $K_{x} \in$ Choice $_{\mathcal{G}}^{x}$ such that $K_{x} \neq s(x)$. Let $s^{*}$ be a function on $\operatorname{dom}\left(s^{*}\right)=\{y \in \operatorname{dom}(s): \exists x \in D(y \leqslant x)\}$ such that $s^{*}(x)=K_{x}$ for each $x \in D$, and $s^{*}(y)=s(y)$ for each $y \in \operatorname{dom}\left(s^{*}\right)-D$. Then $s^{*}$ is a strategy for $\mathcal{G}$ that is backward closed in $M$. Note that by definition, $\mathcal{G}$ is inactive in $\operatorname{dom}\left(s^{*}\right)-D$, from which it follows that

$$
s^{*}(x) \subseteq \operatorname{adh}\left(s^{*}\right) \text { for each } x \in D
$$

For each $y \in \operatorname{dom}\left(s^{*}\right)$, if $y \in D, y \in \operatorname{adm}\left(s^{*}\right)$ by (15); and if $y<x$ for an $x \in D$, $s^{*}(x) \subseteq H_{y}=s^{*}(y)$, and hence $y \in \operatorname{adm}\left(s^{*}\right)$ by (15). It follows that $s^{*}$ is simple in $M$. Consider any $h \in \operatorname{adh}(s) \cap H_{\langle M\rangle}$. Because $s$ is complete along $h$ in $M$, there is a choice point $z \in h \cap \operatorname{dom}\left(s^{*}\right)$ such that $s^{*}(z) \neq s(z)$, and then $h \notin s^{*}(z)$, and hence $h \notin \operatorname{adh}\left(s^{*}\right)$ by definition. It then follows that $\operatorname{adh}(s) \cap a d h\left(s^{*}\right) \cap H_{\langle M\rangle}=\varnothing$, and then by Proposition 5.7, we can extend $s^{*}$ to a complete primary strategy $s^{\prime}$ for $\mathcal{G}$ in $M$, and hence $\operatorname{adh}(s) \cap \operatorname{adh}\left(s^{\prime}\right) \cap H_{\langle M\rangle}=\varnothing$.

\section{Independence}

Recall the condition IA: for each moment $m, \bigcap_{\alpha \in \text { Agent }} f(\alpha) \neq \varnothing$ for each $f \in$ Select $_{m}$, where Select ${ }_{m}$ is the set of all functions each of which assigns each agent $\alpha$ a member of Choice $_{\alpha}^{m}$. This is equivalent to the statement that for each moment $m$, and for all disjoint groups $\mathcal{G}$ and $\mathcal{F}, K \cap K^{\prime} \neq \varnothing$ for all $K \in$ Choice $_{\mathcal{G}}^{m}$ and $K^{\prime} \in$ Choice $_{\mathcal{F}}^{m}$. In our current framework, what each group can do at a moment $m$ are presented as the choices for the group at $m$, which are taken in a good sense to be causally independent of what others can do at $m$. Under the condition IA, nothing $\mathcal{G}$ can do at $m$ may "rule out" anything that $\mathcal{F}$ can do at $m$, where $\mathcal{F}$ and $\mathcal{G}$ are disjoint, nor may anything $\mathcal{G}$ can do at $m$ "force" $\mathcal{F}$ to do one thing at $m$ rather than another. In other words, there is no $K \in$ Choice $_{\mathcal{G}}^{m}$ such that $K \cap K^{\prime}=\varnothing$ for any $K^{\prime} \in$ Choice $_{\mathcal{F}}^{m}$, nor is there any $K \in$ Choice $_{\mathcal{G}}^{m}$ such that $K \subseteq K^{\prime}$ for any $K^{\prime} \in$ Choice $_{\mathcal{F}}^{m}$ if Choice $_{\mathcal{F}}^{m}$ is not a singleton. In general, we may say that for a given partition $\mathbb{A}$ of $H_{m}, \mathbb{A}$ is independent of what $\mathcal{G}$ can do at $m$ iff

$$
\text { for each } K \in \text { Choice }_{\mathcal{G}}^{m}, K \cap H \neq \varnothing \text { for each } H \in \mathbb{A} \text {. }
$$

This notion of independence is a fundamental notion in the decision-theoretical approach to deontic logic, based on which Horty builds his theory of dominance between choices at a point (Horty 2001): A choice $K$ for $\mathcal{G}$ at $m$ dominates another, $K^{\prime}$, if for a partition $\mathbb{A}$ of $H_{m}$, independent of what $\mathcal{G}$ can do at $m, K$ is "better than" $K^{\prime}$ under each condition presented as a member of $\mathbb{A}$. The partition $\mathbb{A}$ of $H_{m}$ that Horty uses is $\operatorname{Choice}_{\overline{\mathcal{G}}}$, which is, as we said above, independent of what $\mathcal{G}$ can do at $m$.

As stated earlier, we want to take the decision-theoretical approach to deontic logic to go beyond single-choice-point situations. To that end, we need a notion of 
independence more general than (16) above, based on which we can build a more general notion of dominance. In this section we provide a preliminary analysis of independence on our current setting.

In our previous discussion of what groups can do through a field $M$, we have identified them as sets of histories that are indistinguishable for the groups in $M$. It would be natural to continue applying such identification in our discussion concerning what one group can do through $M$ being independent of what another group can do through $M$. In the context of deontic logic, nevertheless, it is more convenient to talk about certain background conditions to be independent of certain strategies, rather than being independent of certain sets of histories. So we will identify what groups can do through $M$ with strategies for the groups in $M$, and speak of a set of strategies of which a classification of $H_{\langle M\rangle}$ is independent. ${ }^{21}$

A field $M$ is like a big "point", and a set $S$ of strategies for $\mathcal{G}$ in $M$ is like a set of choices for $\mathcal{G}$ at a point. One might then be attempted to define a classification $\mathbb{A}$ of $H_{\langle M\rangle}$ to be independent of $S$ the same way as (16) for a partition of $H_{m}$ to be independent of Choice $_{\mathcal{G}}^{m}: \mathbb{A}$ is independent of $S$ iff

$$
\text { for each } s \in S, \operatorname{adh}(s) \cap H \neq \varnothing \text { for each } H \in \mathbb{A} \text {. }
$$

This won't do, nevertheless. Suppose that there is a history $h$ passing through $M$ and that $\mathcal{G}$ is a group inactive along $h$ in $M$, which is quite possible. Then, as a consequence of Proposition 8.2, we would have that $\bigcap \mathbb{A}_{M, \mathcal{G}} \neq \varnothing$, and hence for each $s \in C P$-Strategy $y_{\mathcal{G}}^{M}, \operatorname{adh}(s) \cap H \neq \varnothing$ for each $H \in \mathbb{A}_{M, \mathcal{G}}$. Hence, if we define independence as (17), $\mathbb{A}_{M, \mathcal{G}}$ would be independent of $C P$-Strategy ${ }_{\mathcal{G}}^{M}$, which is counter-intuitive. ${ }^{22}$

Let $s$ be a strategy in $M$ and let $H \subseteq H_{\langle M\rangle}$. We say that $s$ guarantees $H$ if $a d h(s) \cap H_{\langle M\rangle} \subseteq H$, and that $s$ excludes $H$ if $\operatorname{adh}(s) \cap H=\varnothing$. One may also be tempted to define a classification $\mathbb{A}$ of $H_{\langle M\rangle}$ to be independent of a set $S$ of strategies just in case

for each $s \in S$ and $H \in \mathbb{A}, s$ neither guarantees nor excludes $H$.

Even though this suggested account is intuitive and simple, a little reflection shows that it would work only in more restricted cases. For example, the trivial classification $\left\{H_{\langle M\rangle}\right\}$ of $H_{\langle M\rangle}$ should be taken to be independent of any set of strategies, but it is not so according to (18) because all strategies guarantee $H_{\langle M\rangle}$. The situation becomes

\footnotetext{
${ }^{21}$ Although identifying what $\mathcal{G}$ can do through $M$ with a strategy $s$ for $\mathcal{G}$ in $M$ is different from identifying it with $a d s(s) \cap H_{\langle M\rangle}$ or with $a_{d o}(s)$, the differences are only technical, not conceptual - they arise from different ways of talking about the same thing.

22 This becomes clearer if we notice that what $\mathcal{G}$ can do through $M$ can be identified with $C P$ Strategy $y_{\mathcal{G}}^{M}$ as well as $\mathbb{A}_{M, \mathcal{G}}$. Under the circumstance described in the main text above, if we were to define independence as (17), what $\mathcal{G}$ can do through $M$ would be independent of what $\mathcal{G}$ can do through $M$.
} 
more complicated once we take into consideration that some groups may be inactive in a proper subset of $M$.

The intuitive idea in our notion of independence is this, which is a slight generalization of (18): Given a classification $\mathbb{A}$ of $H_{\langle M\rangle}$ and a set $S$ of strategies. $\mathbb{A}$ is independent of $S$ if no strategy in $S$ may exclude any member of $\mathbb{A}$, nor may any such strategy guarantee a member of $\mathbb{A}$ without guaranteeing all members of $\mathbb{A}$.

Definition 9.1. Let $M$ be any field, let $\mathbb{A}$ be any classification of $H_{\langle M\rangle}$, and let $S$ be any set of strategies in $M . \mathbb{A}$ is independent of $S$ if the following hold:

(i) for each $s \in S$ and each $H \in \mathbb{A}, a d h(s) \cap H \neq \varnothing$, and

(ii) for each $s \in S$ and each $H \in \mathbb{A}$, $\operatorname{adh}(s) \cap H_{\langle M\rangle} \subseteq H$ only if $\operatorname{adh}(s) \cap H_{\langle M\rangle} \subseteq$ $\bigcap \mathbb{A}$.

It is easy to verify that in the context above, if $\mathbb{A}$ is independent of $S$, so is each subset of $\mathbb{A}$ (as long as it is still a classification of $H_{\langle M\rangle}$ ), which in turn is independent of each subset of $S$. Definition 9.1(ii) may appear wrong because it allows a strategy $s$ in $S$ to guarantee a member $H$ of $\mathbb{A}$, but actually, it allows $s$ to guarantee $H$ only when $s$ guarantees all members of $\mathbb{A}$. Note that if $\mathbb{A}$ is a partition of $H_{\langle M\rangle}$ (not just a classification of $H_{\langle M\rangle}$ ), then Definition 9.1 (ii) amounts to that for each $s \in S$, $a d h(s) \cap H_{\langle M\rangle} \subseteq H$ only if $H=H_{\langle M\rangle}$ (i.e., only if $\mathbb{A}$ is the trivial partition $\left\{H_{\langle M\rangle}\right\}$ ). Note also that if $\mathbb{A}$ is a non-trivial partition of $H_{\langle M\rangle}$, it is then independent of $S$ iff for each $H \in \mathbb{A}$ and each $s \in S$, neither $\operatorname{adh}(s) \cap H=\varnothing$ nor $a d h(s) \cap H_{\langle M\rangle} \subseteq H$. That is to say, this account of independence is a generalization of the account (18) suggested above, and the two accounts work exactly the same if we restrict classifications of $H_{\langle M\rangle}$ to non-trivial partitions of $H_{\langle M\rangle}$. A similar remark can be made about the following easy consequence of Corollary 8.4 (compare it to (18)):

Corollary 9.2. Let $M$ be a field, and let $\mathcal{F}$ be SOL active in $M$. Then for each set $S$ of strategies, $\mathbb{A}_{M, \mathcal{F}}$ is independent of $S$ iff for each $s \in S$ and each $H \in \mathbb{A}_{M, \mathcal{F}}$, $\operatorname{adh}(s) \cap H \neq \varnothing$ and $\operatorname{adh}(s) \cap H_{\langle M\rangle} \nsubseteq H$.

Proof. We only need to assume that $\mathbb{A}_{M, \mathcal{F}}$ is independent of $S$, and show that $\operatorname{adh}(s) \cap$ $H_{\langle M\rangle} \nsubseteq H$ for all $s \in S$ and $H \in \mathbb{A}_{M, \mathcal{F}}$. Suppose for reductio that $\operatorname{adh}(s) \cap$ $H_{\langle M\rangle} \subseteq H$ for an $s \in S$ and an $H \in \mathbb{A}_{M, \mathcal{F}}$. Because $\mathbb{A}_{M, \mathcal{F}}$ is independent of $S$, $\operatorname{adh}(s) \cap H_{\langle M\rangle} \subseteq H^{\prime}$ for all $H^{\prime} \in \mathbb{A}_{M, \mathcal{F}}$, and then, since $\operatorname{adh}(s) \cap H_{\langle M\rangle} \neq \varnothing$, $\bigcap \mathbb{A}_{M, \mathcal{F}} \neq \varnothing$, contrary to Corollary 8.4.

Under the condition of SOL activity, independence is "symmetrical" in the following sense.

Proposition 9.3. Let $M$ be a field, and let $\mathcal{F}$ and $\mathcal{G}$ be disjoint groups that are SOL active in $M$. Then $\mathbb{A}_{M, \mathcal{F}}$ is independent of $C P$-Strategy $y_{\mathcal{G}}^{M}$ iff $\mathbb{A}_{M, \mathcal{G}}$ is independent of $C P$-Strategy $y_{\mathcal{F}}^{M} \cdot 23$

\footnotetext{
${ }^{23} \mathrm{Had}$ we defined independence as a relation between classifications of $H_{\langle M\rangle}$, we would then have that for all disjoint groups $\mathcal{F}$ and $\mathcal{G}$ that are $\mathrm{SOL}$ active in $M, \mathbb{A}_{M, \mathcal{F}}$ is independent of $\mathbb{A}_{M, \mathcal{G}}$ iff $\mathbb{A}_{M, \mathcal{G}}$ is independent of $\mathbb{A}_{M, \mathcal{F}}$.
} 
Proof. Assume that $\mathbb{A}_{M, \mathcal{F}}$ is independent of $C P$-Strategy $y_{\mathcal{G}}^{M}$. Consider any $s \in C P$ Strategy $_{\mathcal{F}}^{M}$ and $H \in \mathbb{A}_{M, \mathcal{G}}$. By Proposition 7.3, there are $s^{\prime} \in C P$-Strategy $y_{\mathcal{G}}^{M}$ and $H^{\prime} \in \mathbb{A}_{M, \mathcal{F}}$ such that $\operatorname{adh}\left(s^{\prime}\right) \cap H_{\langle M\rangle}=H$ and $H^{\prime}=\operatorname{adh}(s) \cap H_{\langle M\rangle}$. By our assumption, $H^{\prime} \cap \operatorname{adh}\left(s^{\prime}\right) \neq \varnothing$, i.e., $\operatorname{adh}(s) \cap H \neq \varnothing$; and by hypothesis and Corollary 8.7, $\operatorname{adh}(s) \cap H_{\langle M\rangle} \nsubseteq H$. It follows from Definition 9.1 that $\mathbb{A}_{M, \mathcal{G}}$ is independent of $C P$-Strategy $y_{\mathcal{F}}^{M}$.

Let $M$ be any field, and let $m$ be any point. $m$ is a starting point of $M$ if $m \in M$ and $m \leqslant x$ for each $x \in M$. A starting point of a field is obviously unique. It is easy to see that if a field $M$ has a starting point, $\operatorname{dom}(s) \cap \operatorname{dom}\left(s^{\prime}\right) \neq \varnothing$ for all backward closed strategies $s$ and $s^{\prime}$ in $M$. We show below that for each $\mathcal{G}$, the classification $\mathbb{A}_{M, \overline{\mathcal{G}}}$ of $H_{\langle M\rangle}$ is independent of what $\mathcal{G}$ can do through $M$, where we identify what $\mathcal{G}$ can do through $M$ with either $C P$-Strategy $y_{\mathcal{G}}^{M}$ or $S$-Strategy $y_{\mathcal{G}}^{M}$, and in the latter case, $M$ needs to have a starting point.

Theorem 9.4. (AC). Let $M$ be any field, and let $\mathcal{G}$ and $\mathcal{F}$ be disjoint groups. Then the following hold:

(i) $\mathbb{A}_{M, \mathcal{F}}$ is independent of $C P$-Strategy $\mathcal{G}_{\mathcal{G}}^{M}$;

(ii) $\mathbb{A}_{M, \mathcal{F}}$ is independent of $S$-Strategy $y_{\mathcal{G}}^{M}$ if $M$ has a starting point;

(iii) $\mathbb{A}_{M, \overline{\mathcal{G}}}$ is independent of $C P$-Strategy $y_{\mathcal{G}}^{M}$, and is independent of $S$-Strategy $y_{\mathcal{G}}^{M}$ if $M$ has a starting point.

Proof. (iii) follows directly from (i) and (ii). Letting $\mathbb{A}=\mathbb{A}_{M, \mathcal{F}}$, we only need to show that Definition 9.1(ii) holds with $S=S$-Strategy ${ }_{\mathcal{G}}^{M}$, and that Definition 9.1(i) holds with $S=C P$-Strategy ${ }_{\mathcal{G}}^{M}$, and with $S=S$-Strategy $y_{\mathcal{G}}^{M}$ if $M$ has a starting point. Let $H \in \mathbb{A}$. By Proposition 7.3, $H=\operatorname{adh}\left(s^{*}\right) \cap H_{\langle M\rangle}$ for an $s^{*} \in C P$-Strategy $y_{\mathcal{F}}^{M}$.

Consider any $s \in S$-Strategy $y_{\mathcal{G}}^{M}$, and suppose that $\operatorname{adh}(s) \cap H_{\langle M\rangle} \subseteq H$. By Proposition 5.7, $\operatorname{adh}(s)=\bigcup_{s^{\prime} \in S^{\prime}} a d h\left(s^{\prime}\right)$ where $S^{\prime}=\left\{s^{\prime} \in C P\right.$-Strategy $y_{\mathcal{G}}^{M}$ : $\left.s \subseteq s^{\prime}\right\}$. Since $\left(\bigcup_{s^{\prime} \in S^{\prime}} a d h\left(s^{\prime}\right)\right) \cap H_{\langle M\rangle} \subseteq \operatorname{adh}\left(s^{*}\right)$, Corollary 8.6 implies that $\left(\bigcup_{s^{\prime} \in S^{\prime}} a d h\left(s^{\prime}\right)\right) \cap H_{\langle M\rangle} \subseteq \operatorname{adh}\left(s^{\prime \prime}\right)$ for each $s^{\prime \prime} \in C P$-Strategy $\mathcal{F}_{\mathcal{F}}$, and hence $\operatorname{adh}(s) \cap H_{\langle M\rangle} \subseteq \operatorname{adh}\left(s^{\prime \prime}\right) \cap H_{\langle M\rangle}$ for each $s^{\prime \prime} \in C P$-Strategy $M_{\mathcal{F}}^{M}$. It then follows from Proposition 7.3 that $a d h(s) \cap H_{\langle M\rangle} \subseteq H^{\prime}$ for each $H^{\prime} \in \mathbb{A}_{M, \mathcal{F}}$. Hence Definition 9.1(ii) holds.

Consider any $s \in S$-Strategy $y_{\mathcal{G}}^{M}$. If $s \in C P$-Strategy $y_{\mathcal{G}}^{M}, \operatorname{dom}(s) \cap \operatorname{dom}\left(s^{*}\right) \neq \varnothing$ by Fact 5.4(iii), and if $s \notin C P$-Strategy $\mathcal{G}_{\mathcal{G}}^{M}$ and $M$ has a starting point, we also have that $\operatorname{dom}(s) \cap \operatorname{dom}\left(s^{*}\right) \neq \varnothing$. Then by Proposition 6.2, $\operatorname{adh}(s) \cap \operatorname{adh}\left(s^{*}\right) \cap H_{\langle M\rangle} \neq \varnothing$, i.e., $\operatorname{adh}(s) \cap H \neq \varnothing$. Hence Definition 9.1(i) holds.

SOL activity and the absence of backward busyness enable us to establish a characterization of independence (Theorem 9.6) in terms of a set-theoretical relation between groups. We begin with a special case.

Proposition 9.5. (AC). Let $M$ be any field, in which $\mathcal{F}$ is not backward busy and all sub-groups of $\mathcal{F}$ are SOL active, except for the empty group. Then for each group $\mathcal{G}$, 
(i) $\mathcal{F} \subseteq \overline{\mathcal{G}}$ iff $\mathbb{A}_{M, \mathcal{F}}$ is independent of $C P$-Strategy ${ }_{\mathcal{G}}^{M}$;

(ii) $\mathcal{F} \subseteq \overline{\mathcal{G}}$ iff $\mathbb{A}_{M, \mathcal{F}}$ is independent of $S$-Strategy $y_{\mathcal{G}}^{M}$, provided that $M$ has a starting point.

Proof. By Theorem 9.4, we only need to assume that $\mathcal{F} \nsubseteq \overline{\mathcal{G}}$, and show that $\mathbb{A}_{M, \mathcal{F}}$ is not independent of $C P$-Strategy $y_{\mathcal{G}}^{M}$ (and hence, not independent of $S$-Strategy $M_{\mathcal{G}}^{M}$ ). By our assumption, $\mathcal{F} \neq \varnothing$. There are two cases.

Case $1, \mathcal{F} \subseteq \mathcal{G}$. Let $s \in C P$-Strategy $y_{\mathcal{G}}^{M}$, let $\mathcal{E}=\mathcal{G}-\mathcal{F}$, and let $s_{\mathcal{F}}$ and $s_{\mathcal{E}}$ be any complete primary extensions of $\left.s\right|_{\mathcal{F}}$ and $\left.s\right|_{\mathcal{E}}$ respectively (see Sect. 6). Then by Proposition $6.4, s=s_{\mathcal{F}} \sqcap s_{\mathcal{E}}$ and $\operatorname{adh}(s)=\operatorname{adh}\left(s_{\mathcal{F}}\right) \cap \operatorname{adh}\left(s_{\mathcal{E}}\right)$, and hence $\operatorname{adh}(s) \cap H_{\langle M\rangle} \subseteq \operatorname{adh}\left(s_{\mathcal{F}}\right) \cap H_{\langle M\rangle} \in \mathbb{A}_{M, \mathcal{F}}$ by Proposition 7.3. Because $\mathcal{F}$ is SOL active in $M$, Corollary 9.2 implies that $\mathbb{A}_{M, \mathcal{F}}$ is not independent of $C P$-Strategy $\mathcal{G}_{\mathcal{G}}^{M} \cdot{ }^{24}$

Case 2, $\mathcal{F} \nsubseteq \mathcal{G}$. Let $\mathcal{E}=\mathcal{F} \cap \mathcal{G}$ and $\mathcal{E}^{*}=\mathcal{F} \cap \overline{\mathcal{G}}$. Then $\mathcal{F}=\mathcal{E} \cup \mathcal{E}^{*}$, and $\mathcal{E} \neq \varnothing \neq \mathcal{E}^{*}$ because $\mathcal{F} \nsubseteq \overline{\mathcal{G}}$ and $\mathcal{F} \nsubseteq \mathcal{G}$. Let $s \in C P$-Strategy $y_{\mathcal{G}}^{M}$. It suffices to show that $\operatorname{adh}(s) \cap H=\varnothing$ for an $H \in \mathbb{A}_{M, \mathcal{F}}$. Letting $s_{\mathcal{E}}$ and $s_{\mathcal{G}-\mathcal{E}}$ be any complete primary extensions of $\left.s\right|_{\mathcal{E}}$ and $\left.s\right|_{\mathcal{G}-\mathcal{E}}$ respectively, we know by Proposition 6.4 that $s=s_{\mathcal{G}-\mathcal{E}} \sqcap s_{\mathcal{E}}$ and

$$
\operatorname{adh}(s)=\operatorname{adh}\left(s_{\mathcal{G}-\mathcal{E}}\right) \cap \operatorname{adh}\left(s_{\mathcal{E}}\right) .
$$

Let $s_{\mathcal{E}^{*}} \in C P$-Strategy $\mathcal{E}^{*}$, and by Fact 5.4 (iii), let $s^{\prime}=s_{\mathcal{E}} \sqcap s_{\mathcal{E}^{*}}$. By Proposition 6.3 (iv, viii, $\mathrm{x}), s^{\prime} \in C P$-Strategy $y_{\mathcal{F}}^{M}$ and $\operatorname{adh}\left(s^{\prime}\right)=\operatorname{adh}\left(s_{\mathcal{E}}\right) \cap \operatorname{adh}\left(s_{\mathcal{E}^{*}}\right)$. Since $\varnothing \neq \mathcal{E} \subseteq \mathcal{F}$, $\mathcal{E}$ is by hypothesis SOL active but not backward busy in $M$, and then by Proposition 8.8 , there is an $s_{\mathcal{E}}^{\prime \prime} \in C P$-Strategy ${ }_{\mathcal{E}}^{M}$ such that

$$
\operatorname{adh}\left(s_{\mathcal{E}}\right) \cap \operatorname{adh}\left(s_{\mathcal{E}}^{\prime \prime}\right) \cap H_{\langle M\rangle}=\varnothing .
$$

Applying Fact 5.4 (iii), we let $s^{\prime \prime}=s_{\mathcal{E}}^{\prime \prime} \sqcap s_{\mathcal{E}^{*}}$. Then by Proposition 6.3(iv, viii, x) again, $s^{\prime \prime} \in C P$-Strategy $y_{\mathcal{F}}^{M}$ and $\operatorname{adh}\left(s^{\prime \prime}\right)=\operatorname{adh}\left(s_{\mathcal{E}}^{\prime \prime}\right) \cap \operatorname{adh}\left(s_{\mathcal{E}}^{*}\right)$, and hence

$$
\operatorname{adh}(s) \cap \operatorname{adh}\left(s^{\prime \prime}\right) \cap H_{\langle M\rangle}=\varnothing
$$

by (19) and (20). Finally, Proposition 7.3 implies that there is an $H \in \mathbb{A}_{M, \mathcal{F}}$ such that $\operatorname{adh}\left(s^{\prime \prime}\right) \cap H_{\langle M\rangle}=H$, and then $\operatorname{adh}(s) \cap H=\varnothing$ by (21).

Now we are ready to establish a general characterization of independence in terms of a set-theoretical relation between groups.

\footnotetext{
${ }^{24}$ When $\mathcal{F} \subseteq \mathcal{G}$, a weaker condition, that $\mathbb{A}_{M, \mathcal{F}}$ is non-trivial, suffices for $\mathbb{A}_{M, \mathcal{F}}$ not to be independent of $C P$-Strategy $y_{\mathcal{G}}^{M}$. In fact, we can show that $\mathbb{A}_{M, \mathcal{F}}$ is trivial if Definition 9.1 (ii) holds with $\mathbb{A}=\mathbb{A}_{M, \mathcal{F}}$ and $S=C P$-Strategy $\mathcal{F}_{\mathcal{F}}^{M}$ : Suppose that Definition 9.1(ii) so holds. Consider any $h \in H_{\langle M\rangle}$. By Proposition 7.3, $h \in \operatorname{adh}(s) \cap H_{\langle M\rangle} \in \mathbb{A}_{M, \mathcal{G}}$ for an $s \in C P$-Strategy $y_{\mathcal{G}}$. The argument in the main text shows that $h \in a d h(s) \cap H_{\langle M\rangle} \subseteq H$ for an $H \in \mathbb{A}_{M, \mathcal{F}}$, and then by our supposition, $h \in \operatorname{adh}(s) \cap H_{\langle M\rangle} \subseteq \bigcap \mathbb{A}_{M, \mathcal{F}}$. It then follows that $H_{\langle M\rangle} \subseteq \bigcap \mathbb{A}_{M, \mathcal{F}}$, and hence $\mathbb{A}_{M, \mathcal{F}}$ is trivial.
} 
Theorem 9.6. (AC). Let $M$ be any field, in which no group is backward busy, let $\mathcal{E}$ be the group of all agents that are inactive in $M$, and let all other agents be SOL active in $M$. Then for all groups $\mathcal{F}$ and $\mathcal{G}$,

(i) $\mathcal{F}-\mathcal{E} \subseteq \overline{\mathcal{G}}$ iff $\mathbb{A}_{M, \mathcal{F}}$ is independent of $C P$-Strategy $y_{\mathcal{G}}^{M}$;

(ii) $\mathcal{F}-\mathcal{E} \subseteq \overline{\mathcal{G}}$ iff $\mathbb{A}_{M, \mathcal{F}}$ is independent of $S$-Strategy $y_{\mathcal{G}}$, provided that $M$ has a starting point.

Proof. Let $\mathcal{F}$ and $\mathcal{G}$ be any groups, and let $\mathcal{F}^{*}=\mathcal{F}-\mathcal{E}$. Then all sub-groups of $\mathcal{F}^{*}$ are by hypothesis SOL active in $M$, except for $\varnothing$, and hence by Proposition 9.5,

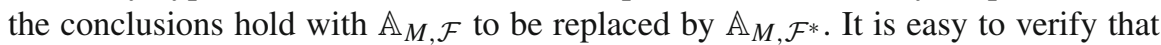
$\mathbb{A}_{M, \mathcal{F} \cap \mathcal{E}}=\left\{H_{\langle M\rangle}\right\}$, and hence $\mathbb{A}_{M, \mathcal{F}^{*}}=\mathbb{A}_{M, \mathcal{F}^{*} \cup(\mathcal{F} \cap \mathcal{E})}=\mathbb{A}_{M, \mathcal{F}}$ by Proposition 7.4.

When dealing with a classification of outcomes bordering a field, we may similarly define its independence of a set of strategies in the following way.

Definition 9.7. Let $M$ be any properly covered field, let $\mathbb{C}$ be any classification of OutcmBdr $r_{M}$, and let $S$ be any set of strategies in $M$. $\mathbb{C}$ is independent of $S$ if the following hold:

(i) for each $s \in S$ and each $U \in \mathbb{C}$, $\operatorname{ado}_{M}(s) \cap U \neq \varnothing$, and

(ii) for each $s \in S$ and each $U \in \mathbb{C}, \operatorname{ado}_{M}(s) \subseteq U$ only if $\operatorname{ado}_{M}(s) \subseteq \bigcap \mathbb{C}$.

The idea in Definition 9.7 is clearly the same as that in Definition 9.1, except that for this new notion of independence to make sense, the strategy field needs to be properly covered. Furthermore, we have the following:

Proposition 9.8. Let $S$ be any set of strategies in a properly covered field $M$, and let $\mathcal{F}$ be any group. Then (i) $\mathbb{C}_{M, \mathcal{F}}$ is independent of $S$ iff (ii) $\mathbb{A}_{M, \mathcal{F}}$ is independent of $S$.

Proof. Suppose that (i) holds. Consider any $s \in S$ and $H \in \mathbb{A}_{M, \mathcal{F}}$. Then $H=\bigcup U$ for a $U \in \mathbb{C}_{M, \mathcal{F}}$ by Proposition 7.9, and then $\operatorname{ado}_{M}(s) \cap U \neq \varnothing$ by (i), and hence $\left(\bigcup \operatorname{ado}_{M}(s)\right) \cap(\bigcup U) \neq \varnothing$. It follows from Proposition 4.6 that $\operatorname{adh}(s) \cap H_{\langle M\rangle} \cap$ $H=\operatorname{adh}(s) \cap H \neq \varnothing$. Hence Definition 9.1(i) holds with $\mathbb{A}=\mathbb{A}_{M, \mathcal{F}}$. Suppose that $\operatorname{adh}(s) \cap H_{\langle M\rangle} \subseteq H(=\bigcup U)$. Then $\operatorname{ado}_{M}(s) \subseteq U$ by Propositions 4.6 and

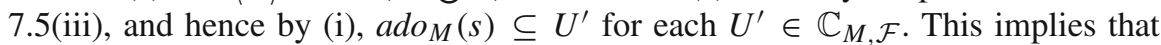
$\operatorname{Uado}_{M}(s) \subseteq \bigcup U^{\prime}$ for each $U^{\prime} \in \mathbb{C}_{M, \mathcal{F}}$, and then by Propositions 4.6 and 7.9, $\operatorname{adh}(s) \cap H_{\langle M\rangle} \subseteq \bigcap \mathbb{A}_{M, \mathcal{F}}$. Hence Definition 9.1(ii) holds with $\mathbb{A}=\mathbb{A}_{M, \mathcal{F}}$, and hence (ii) holds.

Next suppose that (ii) holds. Let $s \in S$ and $U \in \mathbb{C}_{M, \mathcal{F}}$. By Proposition 7.8, $U=\operatorname{ado}_{M}\left(s^{\prime \prime}\right)$ for an $s^{\prime \prime} \in C P$-Strategy $y_{\mathcal{F}}^{M}$, and then, letting $H=\bigcup U$, we know that $H=\operatorname{adh}\left(s^{\prime \prime}\right) \cap H_{\langle M\rangle} \in \mathbb{A}_{M, \mathcal{F}}$ by Propositions 4.6 and 7.3. By (ii), $\operatorname{adh}(s) \cap$ $H \neq \varnothing$, and then $\operatorname{adh}(s) \cap \operatorname{adh}\left(s^{\prime \prime}\right) \cap H_{\langle M\rangle} \neq \varnothing$, and hence by Propositions 4.6 and 3.3, $\bigcup\left(\operatorname{ado}_{M}(s) \cap \operatorname{ado}_{M}\left(s^{\prime \prime}\right)\right)=\bigcup\left(\operatorname{ado}_{M}(s) \cap U\right) \neq \varnothing$, and consequently ado $_{M}(s) \cap U \neq \varnothing$. It follows that Definition 9.7(i) holds with $\mathbb{C}=\mathbb{C}_{M, \mathcal{F}}$. Suppose that $\operatorname{ado}_{M}(s) \subseteq U$. Then by Proposition 4.6, $\operatorname{adh}(s) \cap H_{\langle M\rangle} \subseteq \bigcup U=H$, and hence 
by (ii), $\operatorname{adh}(s) \cap H_{\langle M\rangle} \subseteq H^{*}$ for each $H^{*} \in \mathbb{A}_{M, \mathcal{F}}$. This implies by Proposition 7.5(ii) that $a d h(s) \cap H_{\langle M\rangle} \subseteq \bigcup U^{*}$ for each $U^{*} \in \mathbb{C}_{M, \mathcal{F}}$, and then by Propositions 4.6 and 7.5(iii), $\operatorname{ado}_{M}(s) \subseteq \bigcap \mathbb{C}_{M, \mathcal{F}}$. Hence Definition 9.7(ii) holds with $\mathbb{C}=\mathbb{C}_{M, \mathcal{F}}$, and hence (i) holds.

Applying Proposition 9.8, we can easily establish the following "duals" of Theorems 9.4 and 9.6 .

Theorem 9.9. (AC). Let $M$ be any properly covered field, and let $\mathcal{G}$ and $\mathcal{F}$ be disjoint groups. Then the following hold:

(i) $\mathbb{C}_{M, \mathcal{F}}$ is independent of $C P$-Strategy $\mathcal{G}_{\mathcal{G}}^{M}$;

(ii) $\mathbb{C}_{M, \mathcal{F}}$ is independent of $S$-Strategy $y_{\mathcal{G}}^{M}$ if $M$ has a starting point;

(iii) $\mathbb{C}_{M, \overline{\mathcal{G}}}$ is independent of $C P$-Strategy ${ }_{\mathcal{G}}^{M}$, and is independent of $S$-Strategy $y_{\mathcal{G}}^{M}$ if $M$ has a starting point.

Theorem 9.10. (AC). Let $M$ be any field, in which no group is backward busy, let $\mathcal{E}$ be the group of all agents that are inactive in $M$, and let all other agents be SOL active in $M$. Then for all groups $\mathcal{F}$ and $\mathcal{G}$,

(i) $\mathcal{F}-\mathcal{E} \subseteq \overline{\mathcal{G}}$ iff $\mathbb{C}_{M, \mathcal{F}}$ is independent of $C P$-Strategy $y_{\mathcal{G}}^{M}$

(ii) $\mathcal{F}-\mathcal{E} \subseteq \overline{\mathcal{G}}$ iff $\mathbb{C}_{M, \mathcal{F}}$ is independent of $S$-Strategy $y_{\mathcal{G}}^{M}$, provided that $M$ has a starting point.

The following is an easy consequence of Theorems 9.6 and 9.10 and Definitions 9.1 and 9.7 .

Corollary 9.11. (AC). Let $M$ be any field, in which no group is backward busy, let $\mathcal{E}$ be the group of all agents that are inactive in $M$, and let all other agents be SOL active in $M$. Then for all groups $\mathcal{F}$ and $\mathcal{G}$,

(i) $\mathcal{F}-\mathcal{E} \subseteq \overline{\mathcal{G}}$ iff $\mathbb{A}$ is independent of $C P$-Strategy ${ }_{\mathcal{G}}^{M}$ for each classification $\mathbb{A}$ of $H_{\langle M\rangle}$ such that $\mathbb{A} \subseteq \mathbb{A}_{M, \mathcal{F}}$ iff $\mathbb{C}$ is independent of $C P$-Strategy $y_{\mathcal{G}}^{M}$ for each classification $\mathbb{C}$ of OutcmBdr ${ }_{M}$ such that $\mathbb{C} \subseteq \mathbb{C}_{M, \mathcal{F}}$;

(ii) if $M$ has a starting point, then $\mathcal{F}-\mathcal{E} \subseteq \overline{\mathcal{G}}$ iff $\mathbb{A}$ is independent of $S$-Strategy $y_{\mathcal{G}}^{M}$ for each classification $\mathbb{A}$ of $H_{\langle M\rangle}$ such that $\mathbb{A} \subseteq \mathbb{A}_{M, \mathcal{F}}$ iff $\mathbb{C}$ is independent of S-Strategy $\mathcal{G}_{\mathcal{G}}^{M}$ for each classification $\mathbb{C}$ of OutcmBdr $r_{M}$ such that $\mathbb{C} \subseteq \mathbb{C}_{M, \mathcal{F}}$.

This completes our preliminary study on independence. In order to achieve a general notion of dominance in the current setting, we need to consider some issues involved in independence and the sure-thing principle. We leave those issues to a future study.

Open Access This chapter is distributed under the terms of the Creative Commons Attribution Noncommercial License, which permits any noncommercial use, distribution, and reproduction in any medium, provided the original author(s) and source are credited. 


\section{References}

Belnap, N. 1991. Before refraining: Concepts for agency. Erkenntnis 34: 137-169.

Belnap, N. 1996a. Agents in branching time. In Logic and reality: Essays in pure and applied logic, in memory of Arthur Prior, ed. B.J. Copeland, 239-271. Oxford: Oxford University Press.

Belnap, N. 1996b. Deontic kinematics and austere strategies, ed. P. K. T. Childers and V. Svoboda, Logica '96: Proceedings of the 10th International Symposium, Filsosofia, 21-40.

Belnap, N., and M. Perloff. 1988. Seeing to it that: A canonical form for agentives. Theoria 54: 175-199.

Belnap, N., M. Perloff, and M. Xu. 2001. Facing the future: Agents and choices in our indeterminist world. Oxford: Oxford University Press.

Broersen, J., A. Herzig, and N. Troquard. 2006. A stit-extension of ATL, ed. M. Fisher, 69-81. In Proceedings of Tenth European Conference on Logics in Artificial Intelligence, JELIA 06. Lecture Notes in Artificial Intelligence, vol. 4160. Berlin: Springer.

Brown, M. 2008. Acting, events and actions, ed. R. van der Meyden, and L. van der Torre, 19-33. In Deontic Logic in Computer Science: 9th International Conference, DEON 2008, Luxembourg, Luxembourg, July 2008, Proceedings. Lecture Notes in Artificial Intelligence, vol. 5076. Berlin: Springer.

Horty, J.F. 1989. An alternative stit operator. Manuscript, Department of Philosophy, University of Maryland.

Horty, J.F. 2001. Agency and deontic logic. Oxford: Oxford University Press.

Kooi, B., and A. Tamminga. 2008. Moral conflicts between groups of agents. Journal of Philosophical Logic 37: 1-21.

Müller, T. 2005. On the formal structure of continuous action. In Advances in Modal Logic, vol. 5, ed. R. Schmidt, I. Pratt-Hartmann, M. Reynolds, and H. Wansing, 191-209. London: King's College Publications.

Prior, A. 1967.Past, Present and Future. Oxford: Oxford Univerity Press.

Savage, L. 1954. The foundations of statistics. Second revised edition. New York: Dover Publications.

Tamminga, A. 2013. Deontic logic and strategic games. Erkenntnis 78(1): 183-200.

Thomason, R.H. 1970. Indeterminist time and truth-value gaps. Theoria 36: 264-281.

Thomason, R.H. 1984. Combinations of tense and modality. In Handbook of philosophical logic, vol. 2, ed. D. Gabbay, and F. Guenthner, 135-165. Dordrecht: Reidel Publishing Company.

Thomason, R.H., and J.F. Horty. 1996. Nondeterministic action and dominance: Foundations for planning and qualitative decision. In Proceedings of the Sixth International Conference on Theoretical Aspects of Rationality and Knowledge (TARK-96), vol. 2, 229-250. San Francisco: Morgan Kaufmann Publishers.

von Kutschera, F. 1986. Bewirken. Erkenntnis 24: 253-281.

Xu, M. 1995. Busy choice sequences, refraining formulas and modalities. Studia Logica 54: 267301.

Xu, M. 1997. Causation in branching time (I): Transitions, events and causes. Synthese 112: 137192.

Xu, M. 2010. Combinations of stit and action. Journal of Logic, Language and Information 19(4): 485-503.

Xu, M. 2012. Actions as events. Journal of Philosophical Logic 41: 765-809. 